\title{
11 Nature-Inspired Optimization of Transport in Porous Media
}

Marc-Olivier Coppens ${ }^{1,2 *}$ and Guanghua $\mathrm{Ye}^{2}$

(" Corresponding author: m.coppens@ucl.ac.uk)

${ }^{1}$ Department of Chemical Engineering, University College London, London WC1E 7JE, UK

${ }^{2}$ State Key Laboratory of Chemical Engineering, East China University of Science and Technology, Shanghai 200237, China

\subsection{Introduction}

Transport of molecules across multiple length scales is of great practical importance, from food products and building materials to the recovery, production and distribution of chemicals and energy. Many relevant processes involve porous media; these include catalytic and separation processes, oil and gas recovery, and the delivery of pharmaceuticals. An effective transport system should be scalable, efficient and robust. These properties depend on the multiscale architecture of the transport system, that is, its morphology (shape) and topology (connectivity) at multiple length scales. An optimized transport system boosts production, saves time and cost, and reduces waste. This holds true for the infrastructure for transporting goods and information, as much as for the transport of molecules in porous media. To be optimal, the transport system needs to be suited to serve the other processes in the system, where production or consumption occurs. If these are not properly matched, transport limitations occur. This includes processes involving porous catalysts in chemical engineering, which we focus on in this Chapter, although much of the discussion can be translated to other processes involving porous media as well.

The optimized transport system for such technical applications is not easy to obtain, but we can seek inspiration from biology. Indeed, through billions of years of evolution, plants and animals have acquired highly effective transport systems, crucial to their survival. Although a chemical 
engineering application is different from a biological one in terms of materials (which could be inorganic instead of organic) and operating conditions (which could involve high temperatures and pressures instead of mild, ambient conditions), they share fundamental features. All rely on effectively connecting the action at microscopic scales (of cells, in the case of biology, or active sites, in the case of catalysts) with the overall system (the organism in biology or the reactor in a catalytic process). Robustness and scale independence are important in both instances. For this, they both rely on multiscale architectures, an example of which is illustrated in Fig. 11.1, while the dominant transport mechanism at each scale is governed by physics that are length scale dependent. Based on these common features, it is desirable to seek guidance from nature, to help improve the transport architectures of porous media for engineering applications. Another reason is the ability to design and optimize porous materials for these applications from scratch. This is possible in certain applications, like catalysis or fuel cells, as opposed to applications where transport networks would have to be adapted from existing plans, and are thus more difficult to change, as in city planning or in resource exploration in porous rocks.
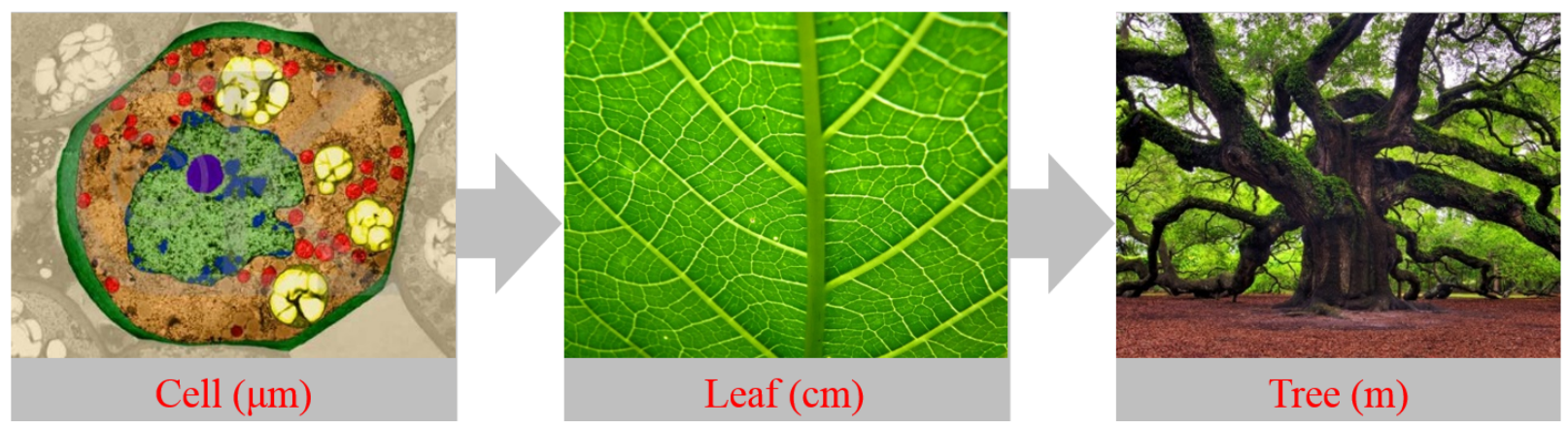

Fig. 11.1. The multiscale architecture of trees. Cells, $10-100 \mu \mathrm{m}$ in size [1], are the basic building blocks (microscale) containing "active sites" for photosynthesis (nanoscale), converting $\mathrm{CO}_{2}$ and water into carbohydrates; leaves, $1-100 \mathrm{~cm}$ in size, function as "porous photo-catalysts" with a veinal architecture for transport (mesoscale); the tree itself, with its water- and nutrient- 
transporting tree-crown, 1-100 $\mathrm{m}$ in size, function as a living "reactor", providing mechanical strength and scalability during growth.

Unlike transport architectures in nature, which were introduced in Part II of this book, transport pathways in artificial porous media are currently not the product of organic evolution. However, they can be optimized by mathematical modeling and computation, which could, for that matter, employ genetic algorithms inspired by evolution. Incredible progress in materials synthesis and manufacturing methods, with increasing control over structure extending down to ever-smaller scales, provides the opportunity to boost the performance of processes employing these materials. To do so effectively, requires guidance from theoretical insights and computational optimization.

In this Chapter, some fundamental features of transport networks in porous media are introduced, and the structure-function relationships in these systems are briefly reviewed to introduce the available "handles" that can be used to manipulate molecular transport and improve the performance of processes that depend on it, like catalysis and molecular separations. These two Sections are presented first, because a good understanding is essential to optimize transport phenomena in engineered systems. Subsequently, the nature-inspired chemical engineering (NICE) approach for transport optimization is introduced, and applications to heterogeneous catalysis and proton exchange membrane (PEM) fuel cells are given to illustrate this methodology.

\subsection{Fundamental features of mass transport phenomena in porous media}

Mass transport in porous media occurs primarily by two mechanisms, namely convective flow and diffusion. In wide pore channels, convective, pressure-driven flow is often the principal 
transport mechanism [2]. In narrower channels, diffusion is the dominant transport mechanism. Self-diffusion is a result of the thermal motion of molecules, while transport diffusion results from a chemical potential gradient; for non-interacting molecules, at sufficiently low pressure, self- and transport diffusivities are the same $[3,4]$. For many processes in chemical engineering and beyond, involving porous catalysts, membranes, building materials and pharmaceutical tablets, for example, diffusion takes place in porous materials containing a hierarchical pore network, and diffusion can be subdivided into molecular diffusion, Knudsen diffusion, surface diffusion, and configurational diffusion, according to the interactions between the molecules and the pore walls [5-7]. Molecular diffusion dominates when the mean free path of a molecule is much smaller than the local pore size, so that the frequency of intermolecular collisions exceeds that of molecule-wall collisions. Knudsen diffusion becomes dominant when molecule-wall collisions are important. Surface diffusion describes the movement of adsorbed molecules along pore wall surfaces, and becomes important for very narrow pores and strongly adsorbed molecules [8]. Configurational diffusion dominates in zeolites and other microporous materials [7,9], in which the effect of pore walls on the movement of molecules is so strong that diffusion is typically an activated process and, therefore, can be well described in terms of a succession of hops. Some state-of-the-art technologies, like interference microscopy (IFM) and IR microscopy (IRM), are now available to record such transport processes experimentally, even in single particles, which is introduced in Chapter 10. In addition, viscous flow plays an important role for transport in porous materials with wide pores, such as porous membranes for microfiltration and ultrafiltration [6].

Depending on the length scale, different transport mechanisms are involved. These different transport mechanisms often take place simultaneously, which complicates the optimization of the 
transport network. Let us take mass transport and reactions in a fixed-bed reactor packed with catalyst pellets as an example to illustrate this multiscale transport, shown in Fig. 11.2. Reactions take place on the so-called "active sites", which are of atomic or nanoscale dimensions, and dispersed on the internal surface of the porous pellets. The geometric and electronic properties of the active sites determine how some species are bound and converted on the catalyst surface. The local physicochemical conditions around these active sites, like the local species concentrations and temperature, affect the local reaction rates and, thus, the catalytic activity and selectivity. This local environment is influenced by the multiscale transport of reactant and product molecules toward and away from these sites, which frequently leads to spatially non-uniform distributions of reactants and products:

(1) Reactants are transported into catalyst pellets from the bulk phase by overcoming external film mass transfer resistance, and subsequently diffuse into the macropore ( $>50 \mathrm{~nm}$ diameter) and mesopore $(2-50 \mathrm{~nm})$ network, where molecular diffusion and Knudsen diffusion dominate. In, for example, the case of zeolites, molecules further diffuse into the micropore network (pores $<2 \mathrm{~nm}$ diameter), where surface diffusion and configurational diffusion become dominant. Simultaneously, molecules adsorb and react on active sites on the pore walls. Products desorbed from the active sites are transported out of the catalyst pellets in the opposite direction. Intrinsically fast reactions may lead to transport limitations, meaning that the resistance to molecular transport dominates the overall rate of the combined process.

(2) The flow of molecules in the fixed-bed reactor removes products that have been transported out of the catalyst pellets, and brings in reactants that enter the catalyst pellets. This leads to a decrease in reactant concentrations and an increase in product concentrations in the direction of 
the flow. Due to this, the boundary conditions at the interface between the catalyst pellets and the bulk flow in the reactor change from reactor inlet to outlet.

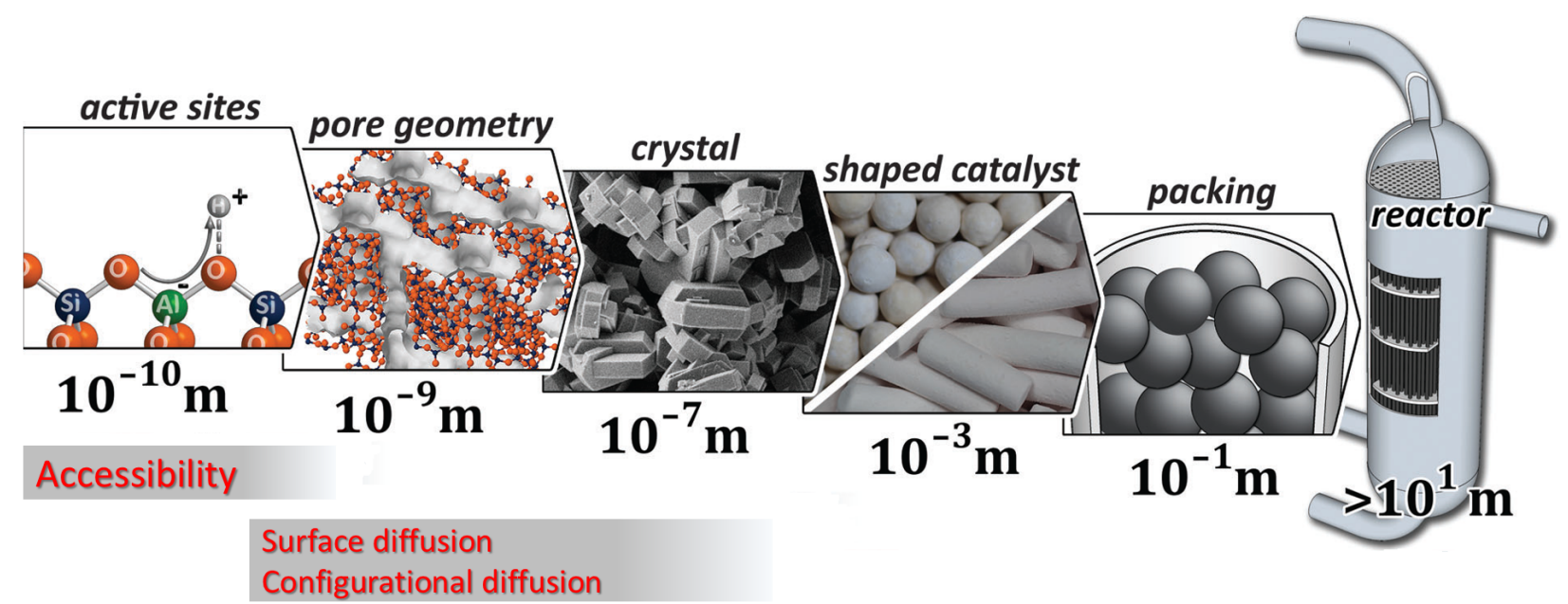

Molecular diffusion

Knudsen diffusion

Flow

Fig. 11.2. Multiscale structure of a fixed bed reactor packed with zeolitic catalyst pellets and the dominant mass transfer mechanisms on different length scales. Reproduced from [10], with permission.

This multiscale transport of molecules is one of the most important, fundamental features for various engineering processes, beyond the example of fixed-bed reactors. In Section 11.6, the example of multiscale transport in PEM fuel cells is also depicted. Already, we can see a parallel with the tree shown in Fig. 11.1, something we will come back to in Section 11.4.

\subsection{Basic description of transport in porous media}

The effective transport properties (permeability for viscous flow and diffusivity for diffusion) depend on the structure of the porous medium, especially the pore size distribution. This provides abundant room for designing porous media with optimized transport properties. To do 
so, it is necessary to formulate relationships between material structure and transport properties. There is a huge literature on this subject, which will not be discussed here in detail. A brief introduction is given in order to aid the understanding of the following sections in this Chapter. Readers can refer to a number of review articles for more details $[5,7,11,12]$.

\subsubsection{Geometrical description of porous media}

Molecular transport networks can be ordered or disordered at different length scales. For example, at the macroscale, fixed bed reactors typically consist of random packings of catalyst particles, in between which the various species flow through a disordered void space. Other reactors employ structured packings, the most common type of which are monolithic structures with parallel channels; the catalytic converter to clean up car exhaust is an example of such a structured packing. In these monoliths, the walls of the channels are porous themselves, or are covered by a catalytic washcoat. At smaller scales, within porous catalysts and other porous materials, molecules diffuse through a network of macro-, meso- and/or micropores. In most amorphous catalyst supports and adsorbents, this pore network is disordered. However, pores can also form a regular network, such as in crystalline zeolites, metal-organic frameworks, and amorphous materials with ordered mesopores [13]. It is easier to model ordered systems and investigate the effects of the regular pore network properties on transport than to model disordered transport networks.

To describe transport in disordered porous media, two types of models are used: continuum models, which treat the porous medium as an effective continuum of reduced permeability or diffusivity, and discrete models, which explicitly account for the pores. Both have been extensively reviewed by Sahimi et al. [14] and Keil [5]. One of the earliest pore models is the parallel pore model proposed by Wheeler [15]. In his model, the pore space is represented by 
parallel pores with mean radius $\bar{r}$ and length $\bar{L}$. The sum of the surface areas of all the parallel pores is set equal to the BET surface area of the particle, and the sum of the pore volumes is set equal to the experimentally determined pore volume of the particle:

$$
\begin{gathered}
\bar{r}=\frac{2 V_{g}}{S_{g}} \sigma(1-\varepsilon) \\
\bar{L}=\sqrt{2} V_{p} / S_{x}
\end{gathered}
$$

where: $V_{g}$ and $V_{p}$ are the specific pore volume and the total volume of the porous particle, respectively; $S_{g}$ and $S_{x}$ are the BET specific internal surface area and the external surface area of the porous particle, respectively; $\sigma$ is the pore wall roughness factor; $\varepsilon$ is the particle porosity. After that, numerous other pore models (including the cylindrical pore model [16], the tortuous pore model [17], the model of Wakao and Smith [18,19], the model of Foster and Butt [20], the grain model [21], and the micro/macropore model [22]) have been proposed to account for more features of real porous materials, such as the tortuosity of the pores or a bidisperse pore size distribution.

Although these early models can describe certain morphological features and, in some cases, account for the pore size distribution, they do not account for the pore network connectivity (topology) and the spatial distribution of the pores. This becomes possible by using pore network models, in which equations for diffusion, adsorption and reaction are explicitly solved, kinetic Monte-Carlo simulations are employed, or various approximations based on statistical physics, like the effective medium approximation or renormalization group theory, are used [23-26].

However, even most pore network representations are still an abstraction of the real porous structure, based on macroscopic data, such as the measured pore size distribution, the porosity 
and the BET surface area. Recently, with the advent of powerful computers and more sophisticated experimental tools, it is becoming possible to digitally reconstruct a real porous structure with increasing accuracy. Some computational methods, including statistical methods (e.g., Monte Carlo method) and process-based methods (e.g., discrete element method) [27,28], have been developed to digitally reconstruct porous materials with high accuracy, as shown in Fig. 11.3a. Cutting-edge experimental technologies, such as X-ray microtomography, directly provide us with three-dimensional (3D) images of porous materials, without even destroying the samples [29,30], as shown in Fig. 11.3b. X-ray nanotomography and electron tomography allow to push the boundaries even further, to unprecedented resolution, although sample sizes are still limited, and care needs to be taken for samples that are anisotropic or macroscopically heterogeneous. In each case, the digitally reconstructed porous structure can be represented by the phase function $f(x)$, which takes the form of a 3D matrix, containing the information of the phase state in each voxel:

$$
f(x)=\left\{\begin{array}{lr}
1 & \text { if } x \text { belongs to pore space } \\
0 & \text { otherwise }
\end{array}\right.
$$

where $x$ is the position vector of a voxel from an arbitrary origin. The digitally reconstructed pore structures can be successfully used to represent rocks [31], membranes [32], fuel cell electrodes [33], porous catalysts [30], fixed beds [29], and many other porous media. Furthermore, these digitally reconstructed pore structures can be reduced to pore networks, using network extraction algorithms, such as the thinning algorithm [34], the medial axis based algorithm [35,36], and the maximal ball algorithm [37,38]. Such digital reconstruction techniques have become a powerful tool for investigating various processes in porous media, especially mass transport. 

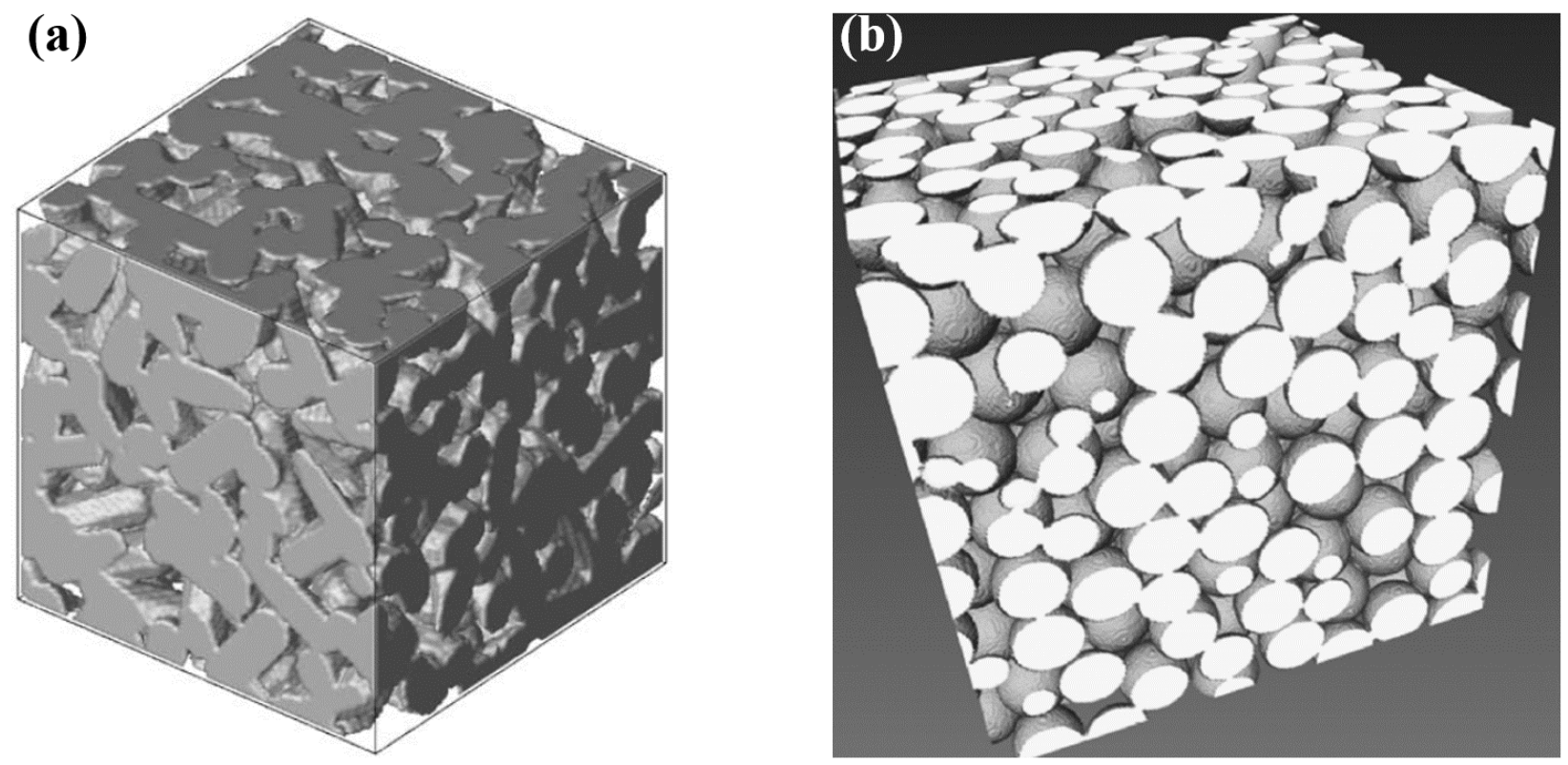

Fig. 11.3. Porous media generated by (a) virtual particle packing and (b) X-ray microtomography scans. From Ref. [30] and Ref. [29], with permission.

\subsubsection{Influence of the structure of porous media on transport properties}

Using the geometrical models briefly introduced in Section 11.3.1, we are able to describe transport in porous media, no matter whether they are ordered or disordered. As a prelude to the optimization studies discussed further on, it is important to understand how the structure of the pore network changes the transport properties. The effects of the geometry of a porous medium on viscous flow and diffusion are briefly recalled. Viscous flow of simple fluids through a single channel or, by extension, a porous medium can be described by Darcy's law, which has been derived from the Navier-Stokes equations via homogenization.

$$
v=-\frac{k}{\eta} \nabla p
$$


Here, $v$ is the so-called Darcy velocity (the average velocity over a volume element containing both fluid and solid matrix), $\eta$ is the viscosity of the fluid, $\nabla p$ is the pressure gradient, and $k$ is the permeability of the (part of a) porous medium under consideration. The permeability for a cylindrical capillary can be calculated using Poiseuille's law [39]:

$$
k=d^{2} / 32
$$

where $d$ is the diameter of the capillary. For a suspension of spheres with diameter $d_{0}$, the permeability can be obtained from the Richardson-Zaki correlation [40]:

$$
k=\left(d_{0}^{2} / 18\right) \varepsilon^{2.7}
$$

For an aggregated bed of spheres with diameter $d_{0}$, the Carman-Kozeny relation can be used to calculate the permeability [6]:

$$
k=\left(d_{0}^{2} / 180\right)\left[\varepsilon^{2} /(1-\varepsilon)^{2}\right]
$$

These are approximations; if more is known about the geometry of the porous medium, the permeability can be estimated more accurately. Structures in which viscous flow occurs, vary in morphology, topology, and randomness, resulting in different equations for the permeability. With advances in techniques to reconstruct the pore space, such as X-ray tomography, and to model flow in porous media, such as Lattice Boltzmann modelling, mesoscopic structural information can be employed to estimate macroscopic viscous flow, including that of complex fluids that can no longer be represented by Darcy's law [29].

Transport diffusion in porous materials, such as porous catalysts and adsorbents, is phenomenologically described by Fick's first law:

$$
\begin{gathered}
J=-D_{e} \nabla c \\
D_{e}=\varepsilon D_{m} / \tau
\end{gathered}
$$


where: $J$ is the diffusion flux; $\nabla c$ is the concentration gradient; $D_{e}$ is the effective diffusivity in the porous medium; $D_{m}$ is the bulk diffusivity; and $\tau$ is the tortuosity, lumping various geometrical (and, possibly, also non-geometrical) factors that affect diffusion in porous materials. Pore size affects the diffusivity through molecule-wall interactions. In micropores, this influence can be so significant that (11.9) is no longer valid and the diffusivity is typically 4-10 orders of magnitude smaller than the one in the bulk phase. The statistical and spatial distributions of pore size also affect the effective diffusivity and tortuosity. Diffusion of molecules tends to be slower when the pore size distribution is wider [41,42]. Tortuosity values as high as 138 have been calculated for a pore network with a connectivity of 3 [43], when the wide and narrow pores of a bimodal pore-size distribution are spatially randomly distributed within the same network; however, this value would be much smaller if a connected network of wide pores surrounds particles with narrow pores, as is more typical in catalyst pellets [43]. The effective diffusivity decreases with decreasing connectivity, but is less dependent on the pore network topology when the connectivity is high enough [42]. The randomness of pore networks also affects the effective diffusivity, especially when the connectivity is low [43-45]. The effective diffusivity of a regular pore network is larger than the one of an irregular pore network, because the diffusion path in the irregular pore network is more tortuous [44].

Amorphous porous materials have a disordered framework, so that their pore walls are not smooth, as is assumed in common cylindrical and spherical pore models, but rough. For many amorphous materials used as catalyst supports and adsorbents, the surface roughness can be described by fractal geometry, similar to natural coastlines [46-52]. Fractals possess scale invariance, that is, they look similar at multiple length scales: magnifying certain parts reveals a structure similar to the whole. 
Benoit Mandelbrot coined the word "fractal," when he discovered that there is a common mathematical language describing such rugged objects, which are infinitely fragmented (like the Cantor set), are lines that are almost nowhere differentiable (like the Koch curve) or are nets with an infinite power law distribution of holes (like the Sierpinski gasket or the Menger sponge) [46]. Each of these objects is strictly self-similar, whatever the magnification. Most importantly, however, what seemed esoteric examples by mathematicians are, in fact, prototypes for similar shapes in nature, like those shown in Fig. 11.5 further on; examples of natural fractals are as diverse as ore distributions, broccoli, clouds, trees, bread, turbulent flow, mountains, or natural coastlines. These are statistically self-similar or self-affine, within a finite range of magnification (self-affine meaning that the similarity under magnification is different along perpendicular directions). Mandelbrot introduced the concept of fractal dimension, $D$; without going into detail, this number conveys, for example, for fractal lines (like the Koch curve or a coastline) the property that such lines have a length that depends on the resolution following a power law, because magnification of parts reveals similar features to the whole. Thus, in the limit of infinite magnification, fractal lines in a plane tend to become infinitely long, yet they still fill less than the plane; thus, they have a dimension that is generally larger than 1 but less than 2: a fractal dimension is usually a broken number. Some fractal lines, like the Peano curve or Brownian motion, are so twisted that they ultimately fill the plane, and have a dimension $D=2$. Fractal surfaces have a dimension larger than 2 , but always lower than 3 , the dimension of the space the surface is contained in.

Many amorphous porous materials have such a fractal, self-similar surface. Hence, the accessible surface area for a molecule depends on its molecular diameter, $\delta$ (effectively, the resolution of observaton), following a power law, $\sim \delta^{2-D}$, where $D$ is the fractal dimension of the surface, a 
number between 2 (for a smooth surface - here the surface is seen to be independent of the size $\delta$ of the molecules used as a measure) and 3 (for a space-filling surface). Clearly, for $D>2$, the surface area becomes larger for smaller probe molecules, indicating that smaller and smaller irregularities alongside the pore walls become accessible, like fjords upon fjords along the Norwegian coastline are accessible to a small boat. The fractal scaling range, within which selfsimilarity holds, is too narrow to significantly affect molecular diffusion, but it has a considerable influence on Knudsen diffusion, because molecule-wall interactions dominate the diffusion behavior. The effect of surface roughness on the Knudsen diffusivity, $D_{K}$, can be approximated by:

$$
D_{K}=D_{K 0} \delta^{D-2}
$$

where $D_{K 0}$ is the Knudsen diffusivity when the pore wall is smooth; a more detailed expression is presented in [51].

\subsection{Nature-inspired engineering approach}

Some of the challenges faced by biological organisms are similar to those we seek to solve for manmade systems. This includes the problem of maintaining efficient operation across length scales, and the related need to efficiently transport molecules across a wide range of length scales. Through billions of years of evolution, biological organisms have developed traits that are particularly effective, especially where these are related to functions essential for survival. Unraveling the fundamental mechanisms underpinning these traits not only helps us to better understand life, and, in medicine, to discover ways to combat disease, but it can also serve as a source of inspiration to solve parallel challenges in technology. 
To do the latter in the most effective manner, it is essential to appreciate both the context and the constraints of the biological model and the engineering application. Properties like remarkable efficiency, adaptability, scalability and resilience in nature may give us pause, when compared to the same properties of manmade systems. Blind imitation of natural features will, however, be highly ineffective. One reason is that the environment of living organisms is often not the same as that of engineering applications, whether it be temperature, pressure or chemical environment. Natural systems are immensely complicated, but not all biological components are necessary in a technical application, because the boundary conditions (available resources, ways to grow or build the system) differ. Also, most solutions need to satisfy multiple objectives simultaneously, while, again, these frequently differ between a biological and a manmade construct. The sources of complexity differ, where constraints of manufacturability, desired time scales, chemical building blocks and scale of operation are often vastly different. Therefore, while the remarkable efficiency of a cell membrane, the agility of a bird or the incredible selectivity of an enzyme may hold valuable information on improving the performance of artificial membranes, aircrafts or catalysts, respectively, purely imitating shape or other all-to-obvious features will rarely lead to a workable, let alone better solution than existing ones.

It is this combination of learning lessons from nature, by seeking to understand the fundamental mechanisms behind desirable features, and applying these mechanisms within the context of a technical application, cognizant of differences in boundary conditions, that we call "natureinspired engineering" or, for chemical engineering applications, nature-inspired chemical engineering (NICE). It differs from biomimicry in its narrow sense, eschewing direct translation of biological features, seeking a deeper understanding of mechanisms and applying these to build a workable technical solution that is acceptable within the constraints that the product or process 
demands (economics, safety, practical applicability, manufacturability, etc.). Thus, our NICE methodology is very much rooted in fundamental physics and chemistry, and combines a holistic approach looking at natural systems with the solution-oriented reductionism and pragmatism of engineering. Our NICE methodology is discussed in a few recent papers [12,53-56], and aims to be a resource for innovation, guiding solutions to challenging problems related to energy, water, health and sustainability in human society.

The complexity of nature is daunting. Its diversity is a fascinating source of beauty, but can also be overwhelming to those seeking to build solutions inspired by nature. Biologists tend to embrace this complexity in all its forms, cataloguing and categorizing it with increasing detail, aiming to be comprehensive. There is value in seeking exceptional behavior that can help us understand evolution as well as reveal rare mechanisms, exceptions to the rule, pushing the boundaries of the biologically achievable - the miracle of the platypus or the bombardier beetle. Such outliers can also inspire out-of-the-box ideas for engineering solutions to technical problems. However, in our NICE approach, in first instance, we look for universal mechanisms that are highly common, and, while biological organisms and systems come in different forms and shapes, the abstraction of physics and mathematical modeling reveals striking similarities.

One of those most striking, universal features in biology is hierarchically structuring, which is also crucial in technology, yet nature is vastly superior in how hierarchical structures are organized, bridging scales from atoms and molecules to organs and organisms, in a way that is essential to their functioning. For example, bone has a hierarchical structure containing seven levels of organization with distinct chemical properties. This allows bone to have unique mechanical properties and transport properties to sustain physiologically important cells, while keeping the overall weight of the bone low [57,58]. Fratzl and Weinkamer [59] illustrate the 
structure-function relation of biological tissues, such as bone, tendon, and wood, at various hierarchical levels, and the importance of this adaptation to fracture healing. Such hierarchical biological structures are a great source of inspiration to materials scientists, seeking to emulate similar properties.

Inspired by the hierarchical structure of the femur, Gustave Eiffel designed the eponymous tower with minimum iron, but strong enough to rise 324 meters into the air. It is important to emphasize that Eiffel, quite obviously, did not copy the entire structure of the bone, but understood that it is the multi-scale balancing of forces in its trabecular structure that holds the secret to combining high strength, flexibility and low weight, as illustrated in Fig. 11.4. The size, shape, and materials used in the construction of the Eiffel tower are different from those of a bone, but it is the hierarchical design with balanced forces at each scale that lends the tower its unique mechanical properties. Scores of similar architectural examples could be cited that are nature-inspired in their design, in the engineering sense, from the work of Gaudí to BuckminsterFuller and Calatrava. The most successful ones marry a nature-inspired design to other properties desired in their application, from functional in the technical sense, to esthetics. 

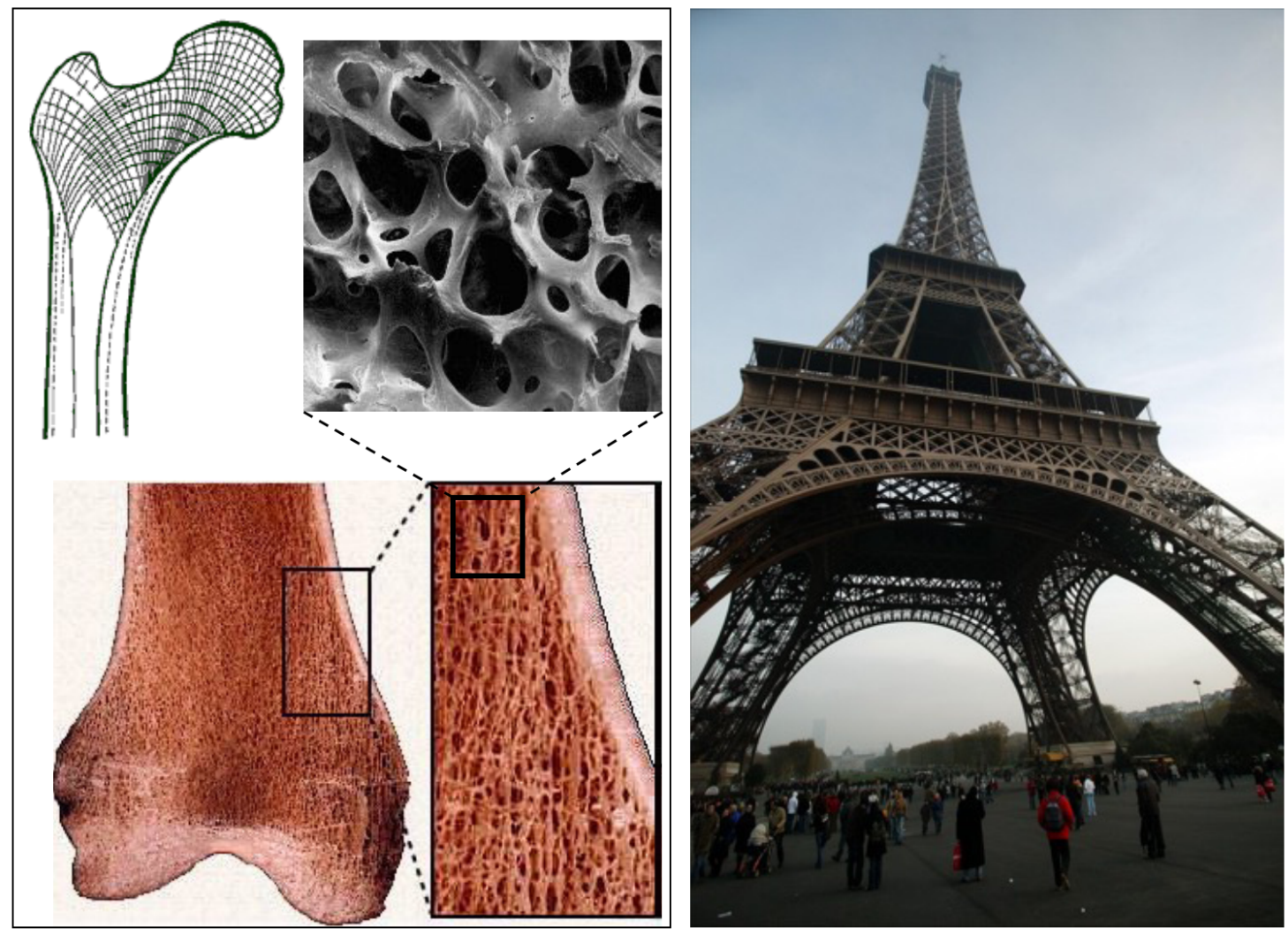

Fig. 11.4. The hierarchical structure of the femur (left [60]) and its inspiration to design the Eiffel tower (right).

Insights into hierarchical structures in biology provide us with a lot of ideas for the optimal design of hierarchically structured materials for processes that rely on efficient mass transport. A hierarchical network is widely adopted in biology to meet the challenge of transporting nutrients toward cells and products, including waste, away from cells through multiple length scales. At macroscopic scales, many of these networks have a fractal, self-similar branching structure, which interpolates between the scale of the organ or entire organism and a minimum length scale, the inner cutoff of the fractal scaling range. Examples are tree crowns (see Fig. 11.5a), the upper respiratory tract of the lungs (see Fig. 11.5b), and the vascular network (see Fig. 11.5c). 
Crucially, the lower bound or inner cutoff of the fractal scaling range also defines a cross-over in the dominant transport mechanism, from flow at large (macroscopic) scales, to diffusion at small (mesoscopic to microscopic) scales.
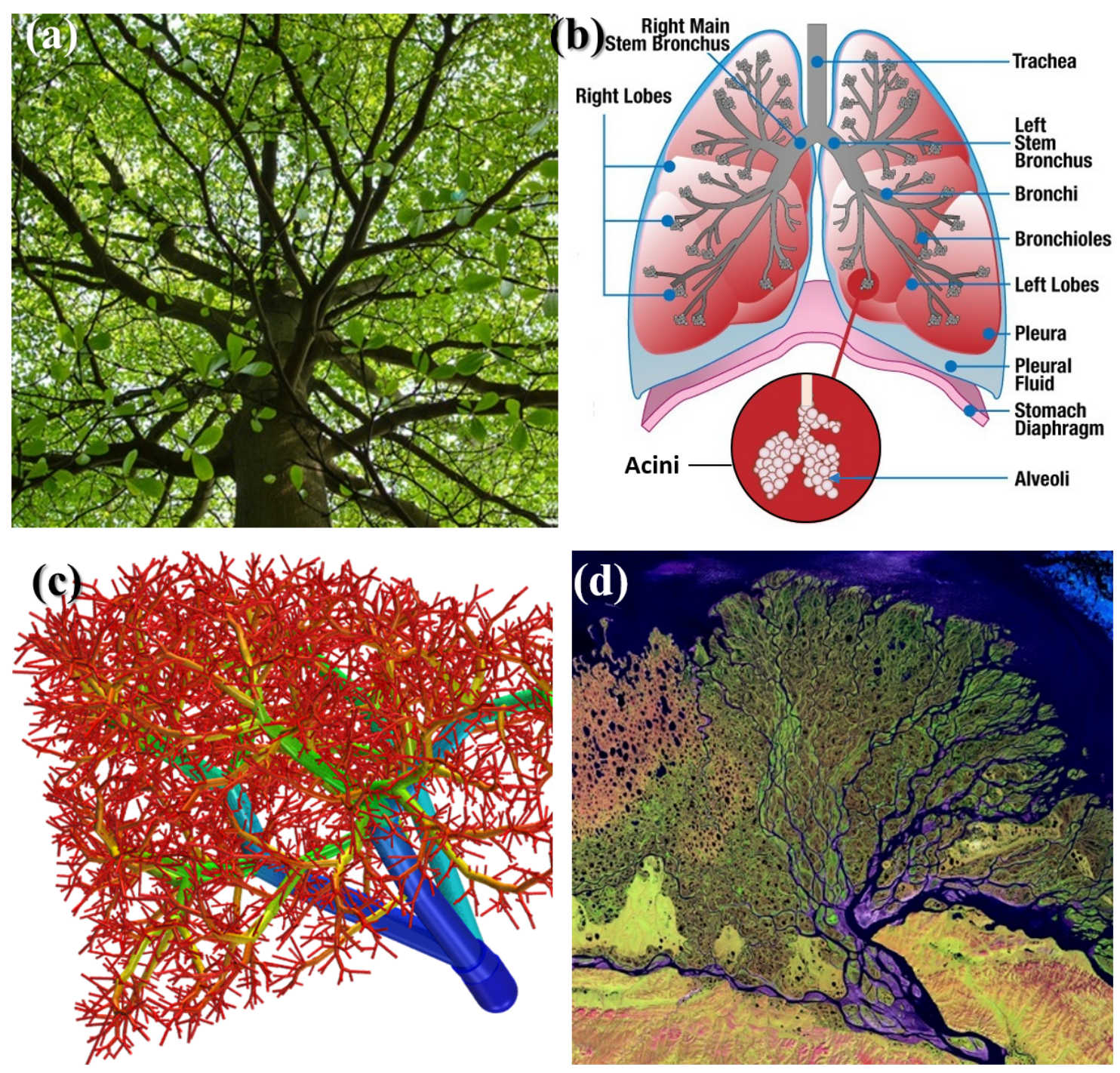

Fig. 11.5. Examples of fractal structures in the nature. (a) Tree crown; (b) lung [61]; (c) a vascular network of the human liver [62]; (d) Lena river delta [63]. 
This is well illustrated by human and other mammalian lungs. The airway tree of a human adult lung repeatedly branches over approximately 23 generations. The upper airway tree is fractal; it consists of 14-16 levels of self-similar branching, counting from the trachea via the bronchi to the terminal bronchioles $[64,65]$. The walls of these upper generations of bronchi are impermeable, and air through the bronchial tree is mainly transported via convective flow. As air flows through the bronchial tree, it gradually slows down from the trachea to the terminal bronchioles. This is because the radius of each branch only gradually decreases from generation to generation. More specifically, at each generation, $\left(r_{\mathrm{p}}\right)^{\Delta}=m\left(r_{\mathrm{d}}\right)^{\Delta}$, where $r_{\mathrm{p}}$ is the radius of the parent branch and $r_{\mathrm{d}}$ is the radius of one of the $m$ daughters; in many cases, $m=2$. The length of the branches decreases similarly from parent to daughter: $\left(l_{\mathrm{p}}\right)^{D}=m\left(l_{\mathrm{d}}\right)^{D}$. Thus, the upper airway tree is a space-filling, self-similar fractal with fractal dimension $D=3$, which also has a diameter exponent $\Delta=3[46,66,67]$. If this diameter exponent, $\Delta$, had been 2 , as it is in most botanical trees (something da Vinci already showed), the flux and the transport velocity would remain constant, because the total cross-sectional area of all daughters remains constant, throughout all branching generations of the tree. However, for the lung, this cross-sectional area progresses with a factor $2^{4 / 3}$ from generation to generation, while the velocity decreases, correspondingly, by a factor $2^{-1 / 3}$. Ultimately, after about 14 generations, air has slowed down so much that diffusional transport, by the random motion of molecules, is as fast as convective transport; any further restriction in channel diameter would make diffusion more rapid than convection. At that point, the Péclet number, $P e$, comparing convective with diffusive transport, crosses over from a value above 1 to one below 1 . It is around this branching generation that the structure of the airway tree changes to one that is very compact, as shown in Fig. 11.5b: air enters the acinar airways, lined by alveoli, where exchange of oxygen and carbon dioxide with the bloodstream 
occurs. Throughout these lower 7-9 space-filling generations of acini, the channel diameter no longer changes much; there would be no advantage to such change, given that, unlike convective flow, diffusive transport by Brownian motion is not affected by the local channel diameter.

In summary, the airway tree acts as a fractal distributor and collector with a self-similar architecture between the macroscopic scale of the trachea to the mesoscopic scale of the bronchioles [68], while the channel size within the acini remains almost constant and the alveoli are uniformly distributed at mesoscopic length scales. A transition in dominant transport mechanism from convection to diffusion, corresponding to $P e \sim 1$, occurs in parallel to this radical change in geometry, and the lower cutoff of the fractal scaling regime defines the cross-over between macroscopic and the mesoscopic length scales. This is a key insight that appears widely valid in biology, where characteristic length scales are tied to cross-overs in function, here exemplified by transport properties. Fractal interpolation between cross-over points bridging the mesoscopic and the macroscopic is common, because it enables preservation of function [56].

Trees show a similar cross-over in hierarchical structure to lungs (Fig. 11.1). The tree crown has a fractal, self-similar branching structure, which distributes water and nutrients, with leaves supported by its branch tips [46]. This self-similar structure is so advantageous in adaptability and scalability that it enables tree crowns to spread tall and wide, without change in structure at the micro- to mesoscale. The branches thicken and the number of branching generations advances with the age of the tree, while the size of the twigs and leaves does not change very much. In deciduous trees, the veinal architecture of leaves transitions from fractal to uniform, again corresponding to a change in dominant transport mechanism from flow to diffusion, where $P e \sim 1$, similar to the case of lungs. 
Thus, a key nature-inspired design principle emerges for artificial hierarchical transport networks in chemical reaction engineering applications and separation processes involving porous materials, namely to combine a fractal geometry at macroscopic scales, and a uniform one at mesoscopic scales, with the reaction, adsorption or exchange process occurring at microscopic scales. This particular hierarchical structure leads to inherent scalability, as the operation is scale independent, but, in addition, the system is also particularly efficient, if not optimal, as we will now discuss.

The ubiquity of transport networks that combine a fractal geometry at larger scales with uniformity at small scales, suggests the importance of understanding the physical reason behind a particular geometry before mimicking it to attempt optimization. Almost a century ago, it was already pointed out by Murray that there is, what he called a "physiological principle of minimum work" $[69,70]$ He proposed to use the concept of "fitness" as a premise for physiological deductions, and hypothesized that physiological organization is such that the energetic cost of operation is minimized. More specifically, he showed that the hierarchical structure of the human vascular network is such that oxygen transport is most efficient. If the blood vessels are too narrow, too much work is needed for blood to flow through, due to high friction. If the vessels are too broad, however, the blood volume is similarly large, which is difficult to sustain as well. Efficiency is a compromise between the factors of work against friction, and the "cost" of upkeep of blood itself, which also requires metabolic energy. Minimizing the total amount of work (per unit of time and per unit of blood volume) as a function of the radius of the blood vessels led Murray to a similar value for the "cost" of blood (energy per unit time and per unit volume) for all arteries and capillaries. Although Murray does 
not use this term, it is very interesting to note that this implies equipartition of energy over the entire system of the vascular network, which is a thermodynamic principle.

We have shown a similar result for the architecture of the lung, and derived it in a different way, using irreversible thermodynamics, that is, second-law energy efficiency or minimization of entropy production [66]. In full agreement with physiological data for the respiratory network, the architecture of the lung is such that the pressure drop over each of the bronchi is the same, and the concentration drop over the acini is the same as well. This implies equipartition of thermodynamic forces over all constituting channels of the respiratory network. The space-filling architecture of the lung, $D=3$, hence, $\left(l_{\mathrm{p}}\right)^{3}=2\left(l_{\mathrm{d}}\right)^{3}$, with also $\Delta=3$, hence $\left(r_{\mathrm{p}}\right)^{3}=2\left(r_{\mathrm{d}}\right)^{3}$, throughout the bronchial tree, leads to minimum power dissipation, given a desired membrane surface area in the acini for exchange with the blood stream. This is a very important principle, which we will use in Section 11.6, when discussing nature-inspired fuel cells.

Underlying this analysis is the observation that we should be very cautious when learning from nature, and blind biomimetics should be avoided. Manmade designs that copy features of biological structures visually or intuitively to achieve similar properties are often referred to as biomimetics or biomimicry. The examples of the lung and the vascular network demonstrate that a physical analysis is necessary to understand the structural features leading to high efficiency and scalability. Straightforward biomimicry might, for example, assume that an infinitely selfsimilar fractal network is best, while our study showed a marked cutoff corresponding to $P e \sim 1$. This adds to the different boundary conditions and context in technological applications, which must be accounted for when using the NICE approach to design and optimize artificial transport systems. We will now illustrate the NICE approach to optimizing transport in porous media, in the case of catalysts and fuel cells. 


\subsection{Nature-inspired optimization of porous catalysts}

Desired properties of porous catalysts include high activity, selectivity, and stability. The geometric and electronic structure of the active sites determines the intrinsic kinetics (microscale), but the pore network structure significantly affects the apparent, effective kinetics (mesoscale), which, in turn, affects overall reactor yields and product distributions (macroscale), via the multiscale hierarchy illustrated in Fig. 11.2. Rational design at the mesoscale has not nearly received as much attention as the microscale, where spectroscopy, quantum chemistry and statistical mechanics have allowed for significant progress. Nevertheless, in a catalyst pellet, the concentrations of certain components might not be uniform, due to their long diffusion path, leading to considerable diffusion resistance. This, in turn, leads to a decreased volume-averaged reaction rate, compared to if the concentrations were uniform throughout the pellet and, therefore, the same to those at the outer surface. The effectiveness factor is defined to quantify the utilization of active sites in a catalyst pellet:

$$
\begin{gathered}
\eta=\frac{\text { rate of reaction with diffusion limitation }}{\text { rate of reaction at outer surface conditions }} \\
\eta=\frac{\int r(C) d V}{r\left(C_{S}\right) V_{t}}
\end{gathered}
$$

where $r(C)$ is the reaction rate per unit volume at a (key) reactant concentration $C$ at any position in the catalyst pellet, $r\left(C_{S}\right)$ is the reaction rate per unit volume at reactant concentration $C_{S}$ at the external surface of the catalyst pellet, and $V_{t}$ is the total volume of the catalyst pellet. A method to determine effectiveness factors by direct experimental inspection via IR imaging was given in Section 10.6. 
Rational design at the microscale must be complemented by similar attention at the mesoscale. Indeed, an important objective is to maximize the effectiveness factor of a desired reaction, without changing the active sites themselves, thus preserving the intrinsic properties. A straightforward method is to shrink the size of the pellet. However, this method is rarely feasible in the chemical industry, because pellet size is typically dictated by reactor engineering requirements, such as pressure drop for fixed-bed reactors (increased for smaller pellets) and the minimum fluidization velocity in fluidized beds (controlled by particle size). Optimal design of the pore network, without affecting the pellet size, is, therefore, necessary to boost the effectiveness factor $[71,72]$.

Here, we can turn to nature for guidance. As illustrated in Fig. 11.2, a leaf bears similarities to a catalyst pellet, catalyzing carbon dioxide and water to sugar and oxygen, for which it is crucial to efficiently transport reactants and products in the leaf. To achieve fast transport, leaves have developed a hierarchical channel system, which we can use as a source of inspiration for the design of hierarchical pore networks in catalysts, as illustrated in Fig. 11.6. A hierarchical pore network in a nanoporous catalyst, like a zeolite, is generated by introducing macro- and mesopores, which act as "highways" for fast transport (see also Section 10.7). However, important questions for the optimal design of these "highways" require an answer: Should they be distributed in a uniform or in a nonuniform way? Should they be of the same size or distributed in size according to an optimal distribution? What should the optimal macro- and mesoporosity be? How sensitive is the design to variations in these textural parameters? Should the optimal pore network be different if deactivation by fouling occurs at the same time? To address these questions, which guide the synthesis of improved catalysts, general features of the optimal pore network in porous catalysts were studied, using computational methods [73-79]. In 
a leaf, as in the lower airway of the lung, the transport network changes from fractal at large scale to uniform at small scales, where diffusion limits transport. The cells are strikingly uniformly distributed amongst the veins in a leaf. The theoretical and computational analysis that now follows does not prove that the leaf has an optimized structure, but we will see that similar features emerge from optimizing a hierarchical porous catalyst.

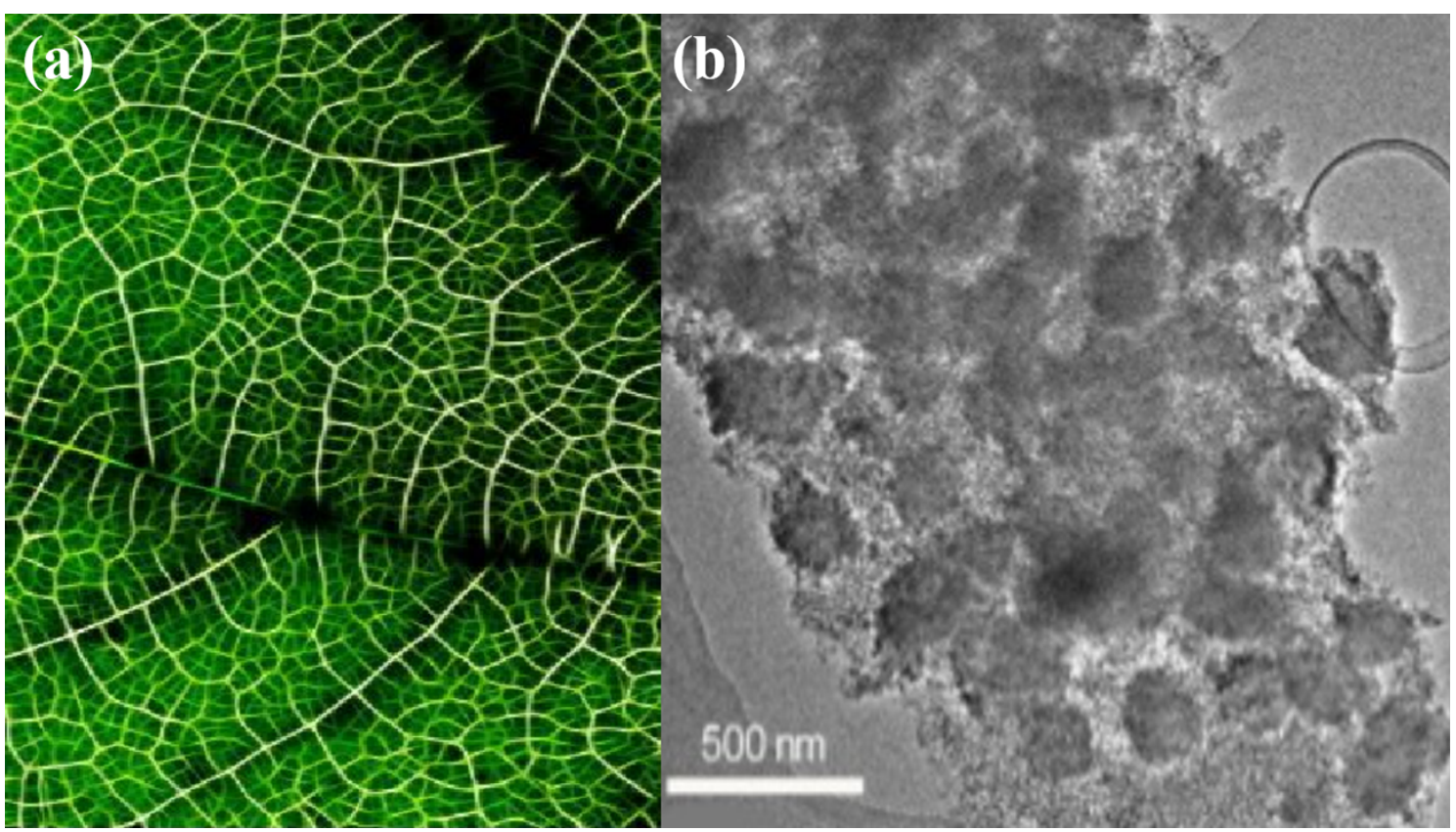

Fig. 11.6. Applying the NICE approach to optimal catalyst pellet design. (a) A leaf has a hierarchical network of veins to quickly transport reactants and products. (b) Inspired by the hierarchical transport network, a ZSM-5 zeolite catalyst was transformed into a hierarchically structured composite with microporous ZSM-5 nanocrystals embedded in a well-connected mesoporous matrix, thus facilitating diffusion. The zeolite composite was synthesized using the route reported in $[80,81]$.

Gheorghiu and Coppens [73] used a two-dimensional model to computationally explore diffusion with first-order, isothermal reaction $(\mathrm{A} \rightarrow \mathrm{B})$ in hierarchically structured catalysts, in 
which a wide-pore network is introduced into a nanoporous catalyst. They found that the catalyst with a fractal-like wide-pore network and broad pore size distribution operates very near optimality, in the sense that the effectiveness factor is maximized. However, the optimum is shallow, and, in these simulations, a constant number of large pores was assumed. This also does not guarantee that the total yield in the pellet is maximized.

Wang et al. [74] relaxed this constraint and compared monodisperse, bidisperse, and bimodal pore networks in a nanostructured catalyst for a first-order, isothermal reaction. For the bidisperse pore network, the large pores all have the same size; in the bimodal pore network, large pores vary in size throughout the pellet, as shown in Fig. 11.7. The computations showed that an optimized bidisperse catalyst could have a yield at least an order of magnitude higher than the one of the monodisperse catalyst (see Fig. 11.7), but also that local variations in pore diameter and porosity of the large pore network, as in general bimodal networks, do not appreciably increase the yield. Transport of molecules results from two diffusion processes, partly in series, partly parallel: (1) diffusion in the large pores penetrating the whole catalyst pellet, (2) local diffusion in the nanoporous "islands" surrounded by the large pores. In the optimal catalysts, the slowest, rate determining process is diffusion in the large pores, because the diffusion path in large pores is orders of magnitude longer than the one in narrow pores. Kärger and Vasenkov [82] reached a similar conclusion experimentally, based on PFG NMR, for catalysts used in fluidized bed catalytic cracking (FCC), namely that diffusion at the (high) reaction temperature in composite faujasite zeolite-containing particles is governed by diffusion in the large pores, rather than in the intracrystalline micropores, despite the intrinsically much smaller diffusivity in the latter. This is because the crystals are so small. Wang et al. [74] also found that the value of the total macro- and mesoporosity is essential, while the distribution of 
the wide (macro-/meso-)pore size is of secondary importance in determining the yield for the optimized hierarchical catalyst. In other words, a spatially uniform, wide pore distribution with uniform pore size (schematically represented by the bidisperse structure in Fig. 11.7) is preferred if the number of wide pores is large enough, while a fractal-like wide pore network may lead to higher yield and effectiveness factor if the number of wide pores is limited. This conclusion is also valid for the optimization of porous adsorbents [83].
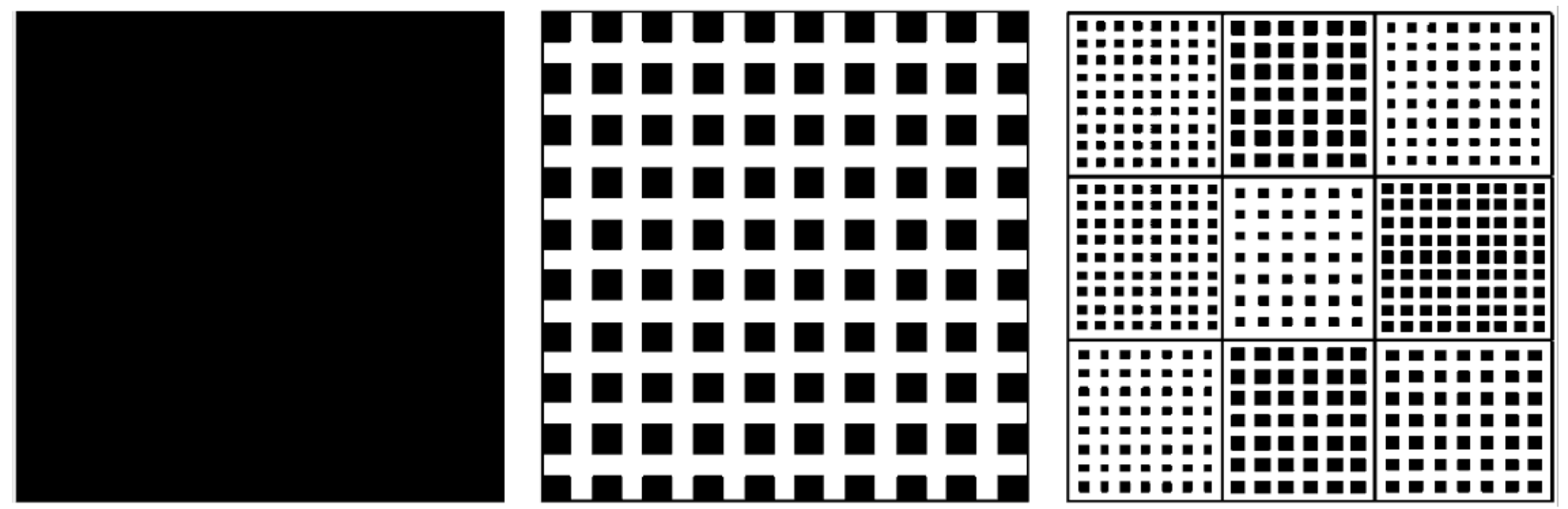

Fig. 11.7. Monodisperse (left), bidisperse (center), and bimodal (right) structures (nanoporous catalytic material: black; large diffusion channels: white). The monodisperse structure has a pore network with only narrow pores. The bidisperse structure has a hierarchical pore network, with narrow nanopores only in the black "islands" of the same size and wide pores of the same size surrounding these "islands". The bimodal structures are assemblies of $N \times N$ bidisperse substructures; in the illustration, $N$ is 3. From [74], with permission.

Introducing macroporosity facilitates molecular transport, on the one hand, and reduces the amount of active catalytic material per unit volume, on the other hand. Hence, there is an optimal macroporosity when the objective is to maximize yield. Johannessen et al. [77] optimized the macroporosity analytically for a periodic bimodal porous catalyst (see Fig. 11.8) using optimal 
control theory and an effective one-dimensional model, with the assumptions of pure molecular diffusion in the large pore channels and first-order, isothermal reaction in the catalyst. For this model catalyst, the macroporosity ( $\varepsilon_{\text {macro }}$ ) can be calculated by:

$$
\varepsilon_{\text {macro }}=\frac{d}{d+w}
$$

where $d$ is the diameter of large channels and $w$ is the channel wall thickness, as shown in the right part of Fig. 11.8. The simulations show that the optimal macroporosity should always be less than 0.5. When channel diameter and channel wall thickness are optimized, concentration gradients are indistinguishable in the $y$ (vertical) direction, which is consistent with the conclusion reached by Wang et al. [74]. Based on this result, a one-dimensional effective (continuum) model was developed; it was shown that this model is almost as accurate as the twodimensional pore network model when optimizing the macroporosity of a bimodal catalyst.

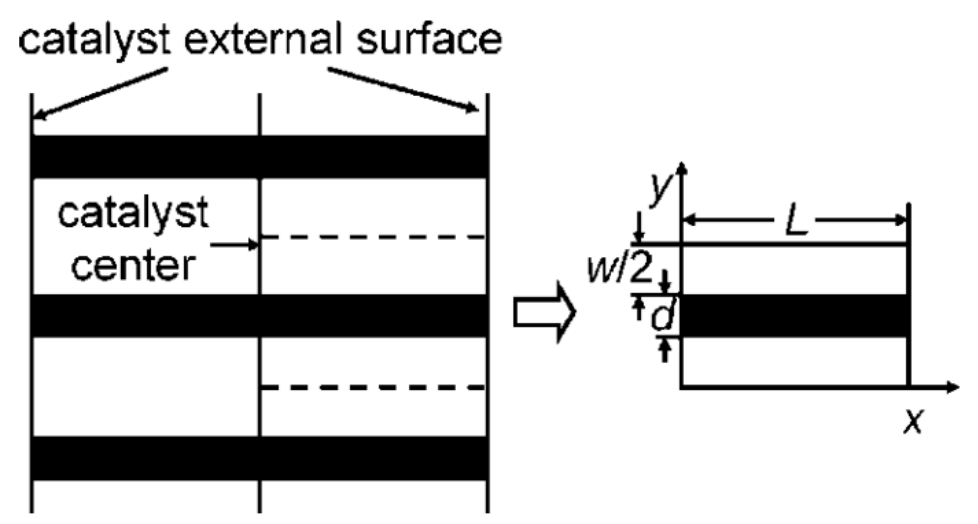

Fig. 11.8. Illustration of the bimodal catalyst (left) and one of its subunits (right). This bimodal catalyst is formed by repeating the subunit in the $y$ direction. The white parts are nanoporous catalytic material; the black parts are large diffusion channels. $L$ is half of the thickness of the catalyst, $w$ is the thickness of the channel wall (i.e., the catalytic material), $d$ is the diameter of the large channels. From [76], with permission. 
The Thiele modulus method can be used to optimize the hierarchically structured porous catalysts. Wang and Coppens [76] defined a generalized distributor (i.e., macropore-based) Thiele modulus $\left(\Phi_{0}\right)$ :

$$
\Phi_{0}=\frac{V}{S} \frac{r\left(C_{0}\right)}{\sqrt{2}}\left[\int_{C_{c}}^{C_{0}} D_{m} r(C) d C\right]^{-1 / 2}
$$

and related $\Phi_{0}$ with the optimal effectiveness factor $\left(\eta_{\text {opt }}\right)$ of the catalyst for a single reaction with general kinetics. In (11.14), $V$ is the volume (3D) or area (2D) of the catalyst pellet; $S$ is the external surface area (3D) or perimeter (2D) of the catalyst pellet; $r$ is the reaction rate; $C_{0}$ is the concentration of a key reactant in the bulk phase; $C_{c}$ is typically assumed to be zero for an irreversible reaction or the concentration in equilibrium for a reversible reaction; $D_{m}$ is the diffusivity in macropores, rather than the effective diffusivity used in the conventional generalized Thiele modulus $(\Phi)$. They found that the $\eta_{\text {opt }}-\Phi_{0}$ relationship (see Fig. 11.9a) is analogous to the classical, universal $\eta-\Phi$ relationship (see Fig. 11.9b), that is, the effectiveness factor $\eta$ is seen to decrease from 1 for a small Thiele modulus (corresponding to high diffusivities and low intrinsic reaction rates) to an inverse proportionality to $\Phi$ at high Thiele modulus. This yields a back-of-envelope approach to design a bimodal catalyst, because $\eta_{\text {opt }}$ can be estimated solely from the value of $\Phi_{0}$ without the need for case-by-case optimizations.

This $\eta_{\text {opt }}-\Phi_{0}$ relation was applied to optimize a mesoporous deNO $\mathrm{x}_{\mathrm{x}}$ catalyst for the pseudo-firstorder, isothermal reaction, $4 \mathrm{NO}+4 \mathrm{NH}_{3}+\mathrm{O}_{2} \rightarrow 4 \mathrm{~N}_{2}+6 \mathrm{H}_{2} \mathrm{O}$, which is used to reduce $\mathrm{NO}_{\mathrm{x}}$ pollutants from power plant emissions [76]. By introducing an optimal macropore network (occupying $20-40 \%$ of the total volume of the catalyst) into the washcoat consisting of the mesoporous deNO $\mathrm{N}_{\mathrm{x}}$ catalyst, its overall activity can be increased by a factor of 1.8-2.8. Wang and 
Coppens [75] also optimized a commercial, mesoporous $\mathrm{Ni} / \mathrm{Al}_{2} \mathrm{O}_{3}$ catalyst for the autothermal reforming of methane by introducing a macropore network. This process produces syngas (a mixture of, mostly, $\mathrm{CO}$ and $\mathrm{H}_{2}$ ), which is the precursor to methanol, ammonia, artificial fuels and more, so it is one of the most important chemical processes. The computations show that the overall activity can be increased by a factor of 1.4-4 by only adjusting macroporosity and macropore size of the bimodal (or macro-mesoporous) catalyst. In addition, a larger macroporosity typically favors a lower $\mathrm{CO} / \mathrm{H}_{2}$ ratio (or a higher selectivity toward hydrogen), which indicates that the macroporosity can be used as a handle to control the $\mathrm{CO} / \mathrm{H}_{2}$ ratio.

(a)

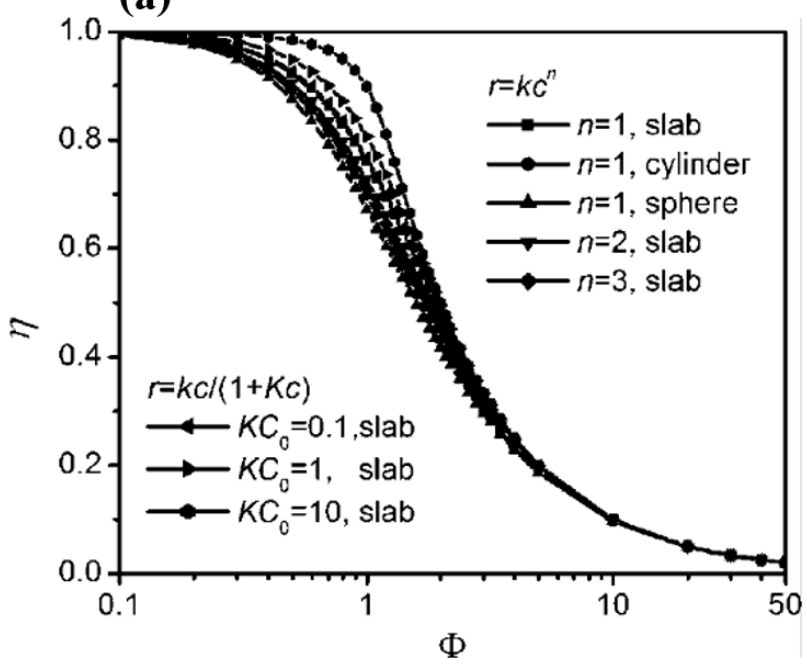

(b)

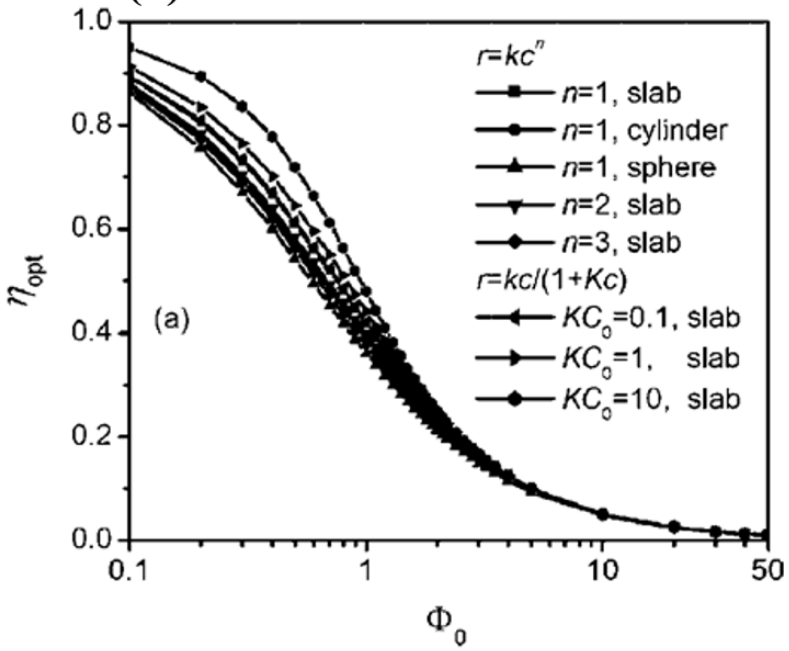

Fig. 11.9. (a) Effectiveness factor of a porous catalyst $(\eta)$ as a function of the generalized Thiele modulus $(\Phi)$ for a single reaction with different reaction kinetics and in catalyst pellets of different shapes. (b) Optimal effectiveness factor of a porous catalyst $\left(\eta_{\text {opt }}\right)$ as a function of the generalized distributor Thiele modulus $\left(\Phi_{0}\right)$ for a single reaction with different reaction kinetics and in catalyst pellets of different shapes. From refer. [76], with permission. 
When optimizing porous catalysts, the sensitivity of the catalyst performance to the structural parameters matters, as this shows how tightly the pore structure should be controlled during synthesis. Wang et al. [74] found that the optimal value of the macroporosity matters the most, while the distribution of large pore size around the optimal large pore size is less important than the size itself. Coppens and Wang [12] investigated how the effectiveness factor reacts to changes in channel diameter $d$ and channel wall thickness $w$ around the optimal values; Fig. 11.10 shows that the loss in effectiveness factor is less than about $5 \%$ within a rather broad region around the optimum. These results are important for the preparation of industrial catalysts, because it is much easier to precisely control the macroporosity, rather than the large pore size.

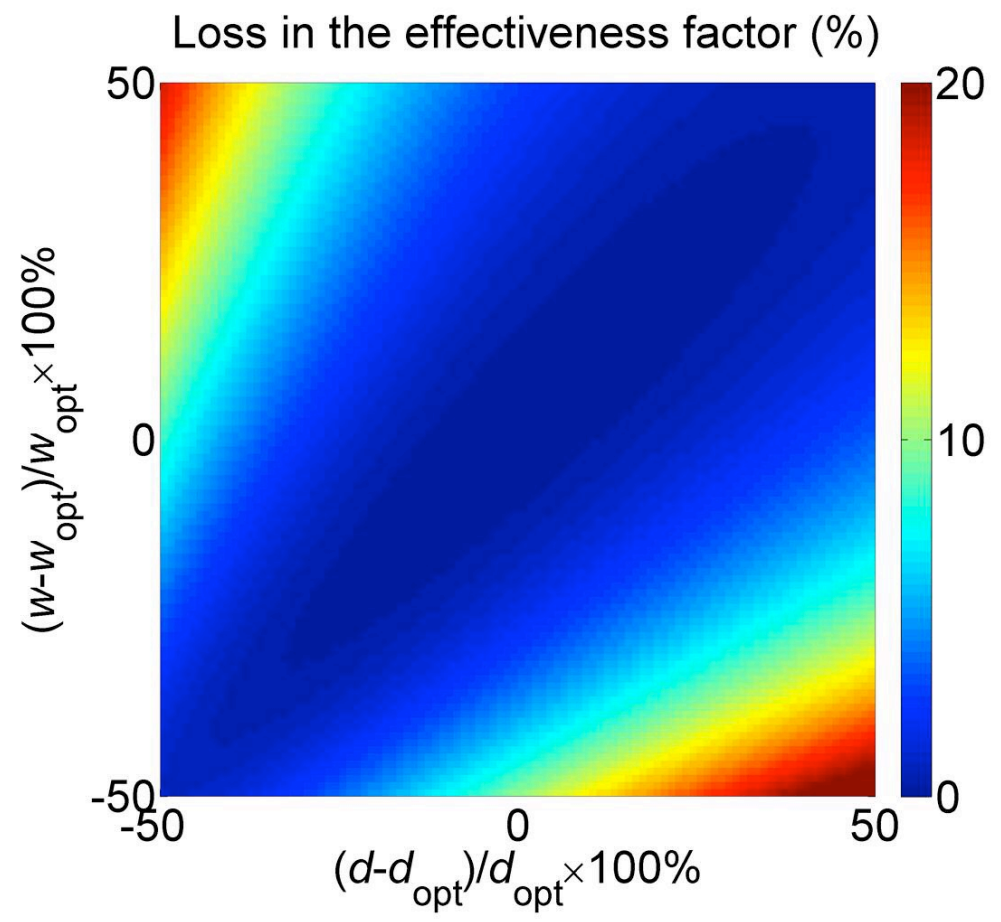

Fig. 11.10. Sensitivity of the effectiveness factor to the variations of channel diameter $d\left(0.5 d_{o p t}{ }^{-}\right.$ $\left.1.5 d_{o p t}\right)$ and channel wall thickness $w\left(0.5 w_{o p t}-1.5 w_{o p t}\right)$, as labeled in Fig. 11.8. The colors indicate the loss in the effectiveness factor (i.e., a percentage of the optimal effectiveness factor). From refer. [12], with permission. 
The performance of a catalyst often changes with time on stream, due to the deactivation of the catalyst by fouling, which covers active sites and blocks pore channels. Deactivation can be mitigated by optimizing the pore network of the catalyst, as suggested by Keil and his colleagues [84,85]. Rao and Coppens [78,79] computationally optimized a mesoporous hydrodemetalation catalyst by introducing an optimal hierarchical pore network, to maximize overall catalytic activity and robustness to deactivation over a given time on stream. This hierarchical pore network structure is illustrated in Fig. 11.11. A random sphere model was used to describe diffusion and reaction in the catalyst pellet. The results show that the lifetime of the hierarchically structured catalyst could be extended by $40 \%$, while using $29 \%$ less catalyst than a non-optimized, purely mesoporous catalyst. Local variations in macroporosity and large pore size only negligibly change the overall yields, which is consistent with the optimization results of the porous catalysts without deactivation [74,77]. Catalytic performance may also be affected by phase change, caused by capillary condensation in the pores. Ye et al. [86] proposed a pore network model to investigate diffusion, phase change, and reaction in a porous catalyst pellet. Hydrogenation of benzene to cyclohexane in the $\mathrm{Pd} / \mathrm{Al}_{2} \mathrm{O}_{3}$ catalyst pellet was selected as a model reaction. Their results show that pore blocking by liquid can significantly affect the performance of the multiphase catalyst, indicating that pore blocking must be accounted for when modelling multiphase reactions. Ye et al. [87] also investigated the influence of pore network structure on the performance of the multiphase catalyst. These structural parameters include pore size distribution, connectivity, pellet size, spatial distribution of pores, and bimodal pore structure. The results show that the performance of the multiphase catalyst is very sensitive to these structural parameters, which indicates that the pore network structure should be well controlled to achieve a desired performance of the porous catalyst for multiphase reactions. 

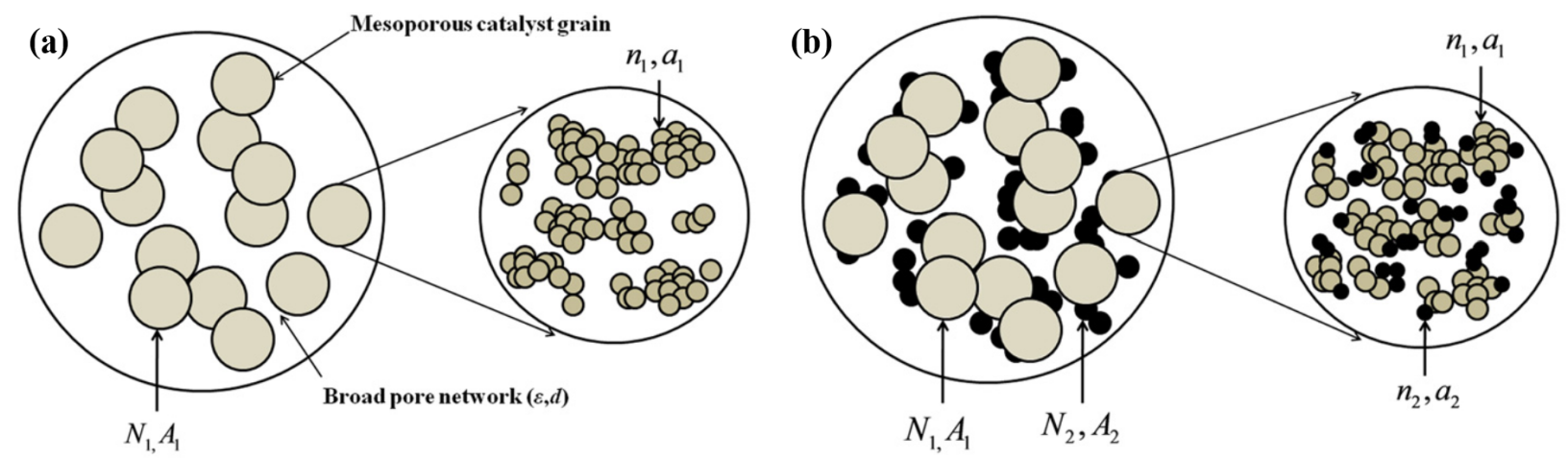

Fig. 11.11. Illustration of the pore structure of the catalyst before and after deactivation. (a) The hierarchically structured catalyst before deactivation is composed of overlapping mesoporous grains separated by a macropore network. Each grain consists of overlapping solid catalyst spheres separated by mesopores. (b) The catalyst after deactivation has a similar hierarchical structure as the one in Fig. 11.11a, but metal sulfide deposits (black spheres) cover the internal surface of the catalyst and can block pores. From [78], with permission.

These studies demonstrate that an appropriate hierarchical catalyst pore network structure can substantially increase catalytic performance, whether it is in terms of activity, selectivity or stability. Within the context of NICE, our conclusions are in striking agreement with models in nature, such as leaves and the alveolar sacs of the lower airways of the lung: A uniform distribution and constant size of "cells" (translated to, e.g., zeolite crystal size) and wide pore channels (translated to macro-/mesopores) leads to maximum performance. The optimal porosity and pore channel size matters, as does the cell/crystal size, avoiding undesired further diffusion limitations within the crystals that would affect the (intrinsic) product distributions and prevent scalability, so important in nature and technology. The benefits are significant and should guide 
synthesis efforts. From a practical viewpoint, the optimum is shallow enough to allow for robust results, as some distribution around the optimum size distribution can be tolerated.

\subsection{Nature-inspired optimization of PEM fuel cells}

Proton exchange membrane (PEM) fuel cells are devices that convert chemical energy into electricity by electro-catalytic oxidation, at the anode, of hydrogen to protons, which diffuse through a membrane and electro-catalytically reduce, at the cathode, oxygen to water. Electrons produced at the anode move through an external circuit (where they are used to power a device) to the cathode, where they are consumed. Rather than direct combustion of hydrogen, the electro-catalytic route avoids Carnot's thermodynamic efficiency limit, thus, while more complicated, is potentially much more efficient, even at low temperatures. A PEM fuel cell consists of electrodes (anode and cathode), catalysts, proton exchange membrane, and gas diffusion layers for gas distribution on both sides of the electrodes. Since the average electric power from a single PEM fuel cell is limited to around $0.5 \mathrm{~W} / \mathrm{cm}^{2}$ [88], several cells must be stacked and bipolar plates are used to connect these cells, in order to achieve the desired power output in applications. During discharge, hydrogen (oxygen) are distributed over the anode (cathode) of the PEM fuel cell through the flow channels on bipolar plates, and then diffuse through the anode (cathode) gas diffusion layer and porous catalyst layer (often, Pt/carbon) before reaching the Pt active sites, where the reactions occur. At the same time, the product, water, is transported through the cathode catalyst and gas diffusion layer, to be collected and removed through the flow channels on the bipolar plates. Severe mass transfer limitations can cause rapid loss of voltage under high loads and significantly reduce power output [89]. Condensed water can clog the pores, but sufficient humidity of the membrane is necessary for the proton exchange to occur. Water management and alleviating, in particular, oxygen mass 
transfer limitations at the cathode is of great importance in PEM fuel cell design. Such problems have persisted over many decades. Can we turn to nature for inspiration in tackling them and redesign PEM fuel cells? We will discuss one aspect of this problem, taking the lung as a source of inspiration.

The required transport systems in PEM fuel cells and in lungs share some fundamental features: a hierarchy of transport channels is used, and dominant transport mechanisms include flow and diffusion. Hence, it is worthwhile to learn from lungs to guide the optimization of transport in PEM fuel cells, which is illustrated in Fig. 11.12. As mentioned in Section 11.4, the upper respiratory tract (from trachea to bronchioles) has a self-similar, fractal architecture in which flow dominates. This fractal architecture connects the microscopic elements (i.e., the acini of the lung) to a single macroscopic element (i.e., the trachea of the lung) via equal hydraulic path lengths, leading to equal transport rates and minimized entropy production while breathing. Besides, this fractal architecture can be extended by simply adding a branching generation, without changing the microscopic building units (i.e., the acini). In the acini of the lung, transport of molecules is dominated by diffusion via the cell walls. Cell size is remarkably constant across mammals, in spite of considerable differences in size between organisms. As discussed, these fundamental properties of the hierarchical structure of the lung are tied to scalability and efficiency of the lung as a gas distributor and collector [66], and so can be utilized to design PEM fuel cells.

Inspired by the lung, a design was proposed to improve the energy efficiency and save the amount of expensive catalytic material in a PEM fuel cell [90]. In this design, the flow channels of a bipolar plate and the pore network architecture of a catalyst layer are optimized. The two parts can be decoupled and subsequently combined. To optimize the flow channel, criteria for 
minimum entropy production should be satisfied; to optimize the pore network structure, an optimized macroporosity should be introduced; both parts are ideally matched when $P e \sim 1$ at the interface, as in the lung.

In the rest of this Section, some examples of biomimetic and nature-inspired designs of flow channels of a bipolar channel are given and compared. An extension to electro-catalysis of the methodology discussed in Section 11.5 is used to optimize the design of the catalyst layer.

\section{Fuel cell}
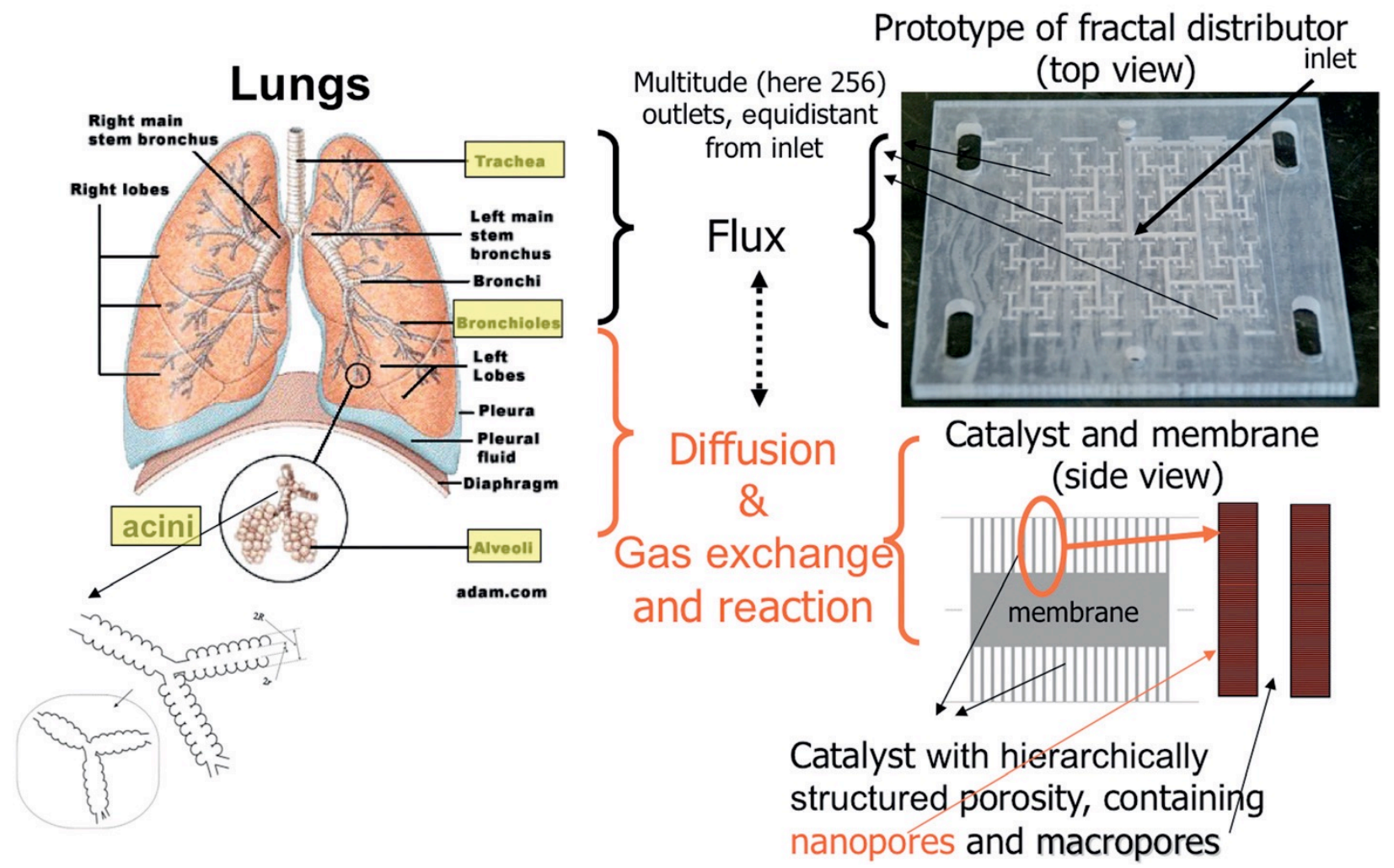

Fig. 11.12. Applying the NICE approach to the optimal design of PEM fuel cells. The hierarchical transport network of the lung, transitioning from fractal to uniform (left) inspired the design of a fractal distributor as bipolar plate (right top) and hierarchically structured nanoporous catalyst with uniformly distributed macropores (right bottom). From [55], with permission. 
Some biomimetic designs of the flow channel pattern have been proposed to improve the flow of reactants and water in a PEM fuel cell [91-93]. Some designs combine the typically used serpentine (snake-like) and interdigitated patterns to form a "leaf-inspired" or "lung-inspired" channel pattern [91,92], as shown in Fig. 11.13. The computations show that the leaf and lung flow channel patterns have a lower pressure drop and a more uniform pressure distribution, compared to the commercial serpentine and interdigitated designs. Experimental studies $[91,92]$ of these biomimetic designs show that the overall fuel cell performance can be increased by $30 \%$. These biomimetic designs are important contributions to the improved design of PEM fuel cells, however, they only mimic certain natural features, without using the rigorous criteria behind the effectiveness of transport in leaves and lungs. Hence, they are essentially empirical, similarities with biology are superficial, and there is no reason for them to be optimal.

(a)

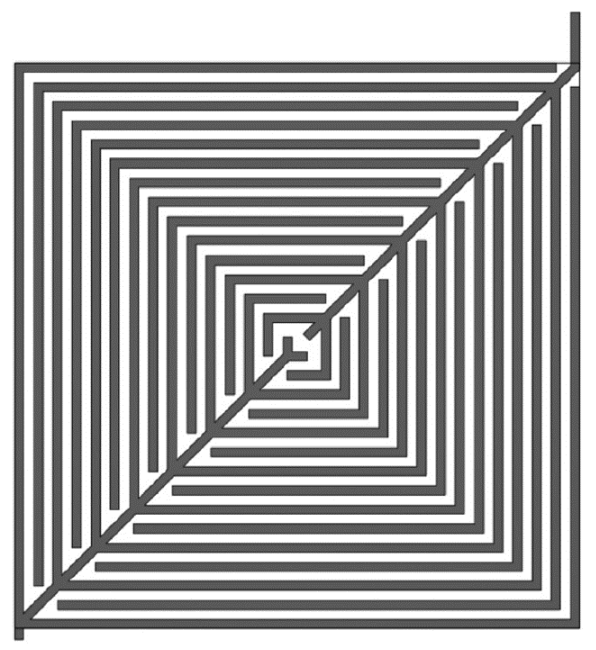

(b)

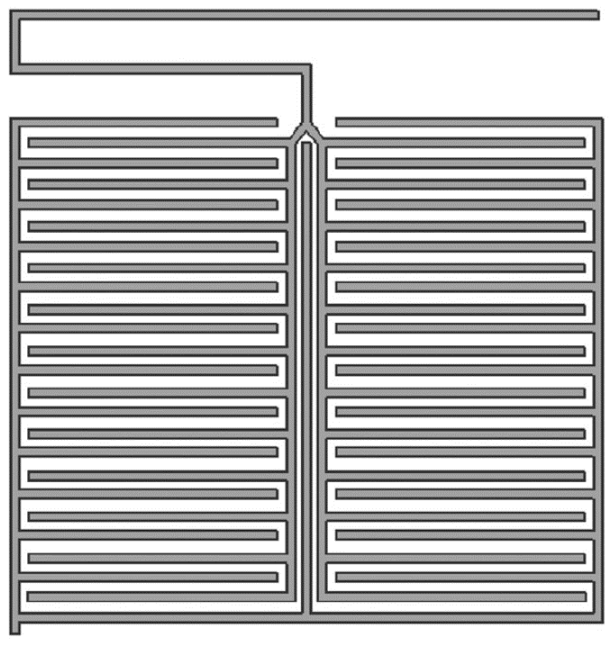

Fig. 11.13. (a) Leaf-inspired and (b) lung-inspired, biomimetic flow channel patterns. The inlet is at the top and the outlet is at the bottom. From [91], with permission.

On the contrary, nature-inspired designs of the flow channels rely on fundamental properties of pulmonary architecture and theories, such as Murray's law [69,70]. A first step is to build flow 
channels into a fractal-like structure, just like the upper respiratory tract of the lung. Fig. 11.14 shows a two-dimensional fractal distributor as bipolar plate, which can be built by rapid prototyping (Fig. 11.12). Reactants enter this distributor through a single inlet, flow through the branching channels, and eventually exit the distributor through a square array of outlets, which have the same hydraulic distance from the inlet. The diameter of the channels gradually changes, following a power law with exponent, $\Delta$, as discussed in Section 11.4. In fractal distributor networks in nature, this exponent is different for botanical trees $(\Delta=2)[46,94,95]$, arteries ( $\Delta=2.7)[46,96]$ and lungs $(\Delta=3)[46,96]$, because the function and transport mechanism in these natural distribution systems differ. Murray's law, where $\Delta=3$, leads to the extraordinary efficiency of the lung. Ramos-Alvarado et al. [97] computationally compared the designs of fractal distributors with 16, 64, and 256 outlets. The fractal distributor with 256 outlets enhanced power generation by $200 \%$ and $50 \%$ over the ones with 16 and 64 outlets, respectively, because flow distribution was more uniform and the pressure drop was lower. Our own work has used $\Delta=3$ in a design that includes a number of branching generations guided by the boundary condition, $P e \sim 1$, thus convective transport out of the last generation matches diffusion in the gas diffusion layer and the catalyst layer adjoining the bipolar plates - similar to the lung (Fig 11.12). 


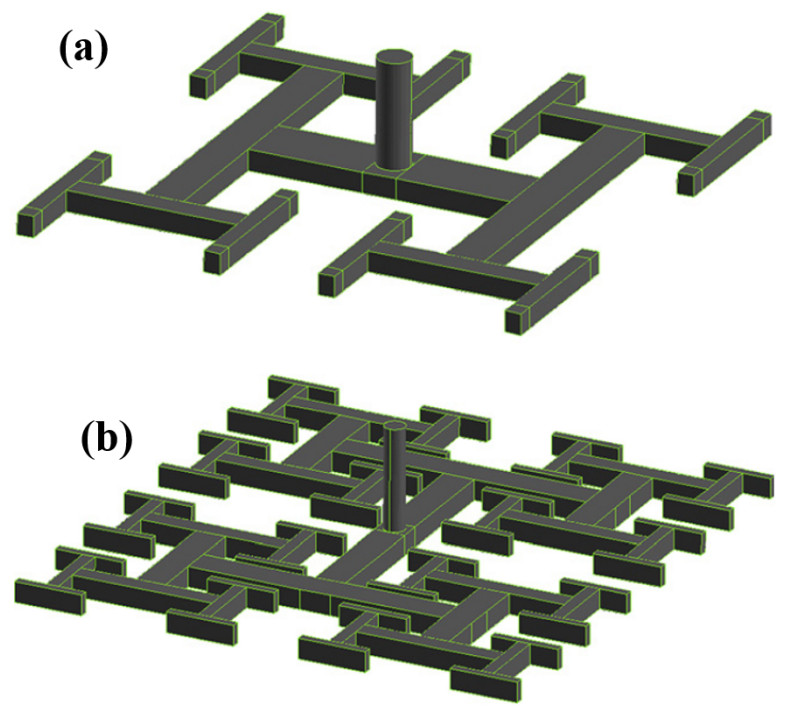

(c)

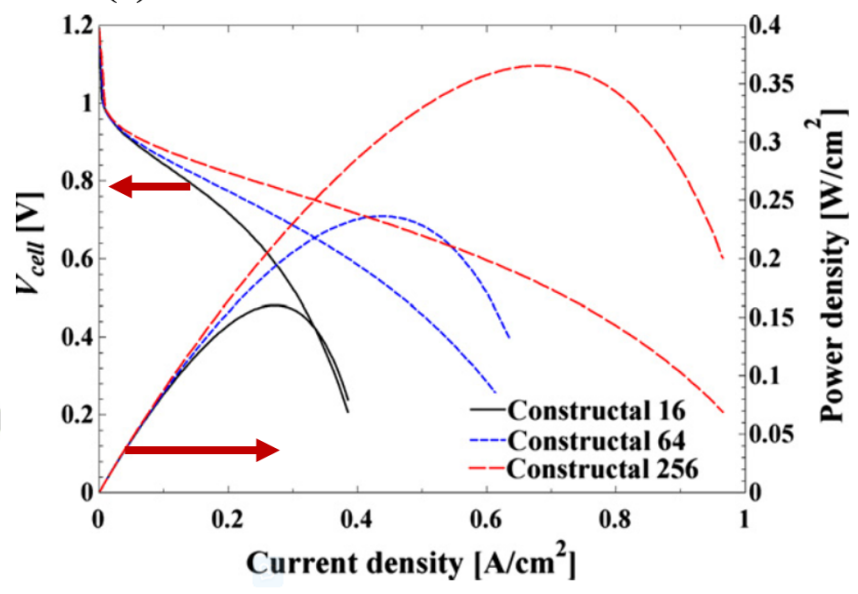

Fig. 11.14. Fractal flow distributors with single inlet and (a) 16 and (b) 64 outlets. (c) Polarization curves (that is, voltage as a function of current density) and power density (product of voltage and current density) of PEM fuel cells for three fractal (here, called constructal) distributors as bipolar plates. The legend "Constructal $N$ " refers to a distributor with $N$ outlets. From [97], with permission.

The inefficient usage of expensive platinum catalyst caused by diffusion limitations not only adds to the total cost, but also decreases the power output. Marquis and Coppens [98] computationally optimized the microstructure by adjusting the platinum loading, platinum-tocarbon ratio, and catalyst layer void fraction. The results show that the optimization of catalyst microstructure can increase platinum utilization 30-fold over existing catalyst layer designs while maintaining power densities over $0.35 \mathrm{~W} / \mathrm{cm}^{2}$. An optimal large pore network should thus be introduced into the catalyst layer to further increase performance, similar to the results obtained in Section 11.5. 


\subsection{Conclusions}

This Chapter discussed a nature-inspired (chemical) engineering (NICE) approach to optimize mass transport, and illustrated it via a few examples relevant to chemical engineering, for catalytic systems employing porous media. In technology, as well as in nature, efficiently transporting molecules over multiple length scales, while maintaining scale-independent results, is of great importance. In each case, the performance of the transport systems is significantly affected by their structure over different length scales, which provides abundant room to optimize transport through manipulating the multiscale structure, such as transport channel size and distribution. Meanwhile, a fundamentally rooted methodology is still required to rationally design these transport systems for technological applications. Trees and mammalian lungs have evolved a hierarchical channel network for transport, which is efficient, robust, and scalable. At the macroscale, where flow dominates, the channel network is a self-similar fractal; at meso- to microscales, where diffusion dominates, the channel size becomes almost uniform. That these structural features are intertwined with functional optimality is a powerful basis for rational, nature-inspired design, beyond biomimicry by superficial imitation. We illustrated this for porous catalysts and PEM fuel cells.

Inspired by hierarchical diffusion networks in biology, an optimal large pore network can be introduced into nanoporous catalysts to maximize the usage of the catalyst, as well as overall yield. Computational and analytical studies indicate that an optimal hierarchically structured catalyst contains uniformly distributed wide pores in between nanoporous catalyst grains; the optimal macro/mesoporosity matters more than the optimal macro/mesopore size, and some distribution around the optimum is allowed, hence the result is robust. The same conclusions hold, irrespective of the reaction kinetics, and such a structure mitigates effects of deactivation 
by fouling. Learning from the fractal architecture of lungs and trees for fast transport across length scales where transport occurs by flow, bipolar plates with a fractal geometry and employing Murray's law were designed to improve the performance of PEM fuel cells, boosting their power output.

Rapid progress in synthesis and manufacturing technologies, from nanomaterials synthesis and microtemplating methods to additive manufacturing and micro-machining, increasingly allow to put theoretically optimized, three-dimensional, hierarchical architectures of porous materials and flow distribution networks into practice. Practical implementation of optimal transport networks, guided by the nature-inspired engineering, NICE, approach, is no longer a distant dream.

\section{References:}

[1] http://www.sciencephoto.com/media/801282/view (n.d.).

[2] T. L. Bergman, A. S. Lavine, F. P. Incropera, and D. P. DeWitt, Fundamentals of Heat and Mass Transfer, 7th ed. (John Wiley \& Sons, Inc., New York, 2011).

[3] D. D. Do, Adsorption Analysis :equilibria and Kinetics (Imperial College Press, London, 1998).

[4] J. Kärger, D. M. Ruthven, and D. N. Theodorou, Diffusion in Nanoporous Materials (Wiley - VCH, Weinheim, 2012).

[5] F. Keil, Catal. Today 53, 245 (1999).

[6] R. Krishna and J. A. Wesselingh, Chem. Eng. Sci. 52, 861 (1997).

[7] R. Krishna, Chem. Soc. Rev. 41, 3099 (2012).

[8] I. Medved and R. Černý, Microporous Mesoporous Mater. 142, 405 (2011). 
[9] J. Kärger, ChemPhysChem 16, 24 (2015).

[10] W. Schwieger, A. G. Machoke, T. Weissenberger, A. Inayat, T. Selvam, M. Klumpp, and A. Inayat, Chem. Soc. Rev. 45, 3353 (2016).

[11] F. J. Keil, Chem. Eng. Sci. 51, 1543 (1996).

[12] M.-O. Coppens and G. Wang, in Des. Heterog. Catal., edited by U. Ozkan (John Wiley \& Sons, Inc., New York, 2009), pp. 25-58.

[13] M.-O. Coppens, in Struct. Catal. React., edited by A. Cybulski and J. A. Moulijn, 2nd ed. (CRC Press, Boca Raton, 2005), pp. 779-805.

[14] M. Sahimi, G. R. Gavalas, and T. T. Tsotsis, Chem. Eng. Sci. 45, 1443 (1990).

[15] A. Wheeler, Adv. Catal. 3, 249 (1951).

[16] M. F. Johnson and W. E. Stewart, J. Catal. 4, 248 (1965).

[17] N. Epstein, Chem. Eng. Sci. 44, 777 (1989).

[18] N. Wakao and J. M. Smith, Ind. Eng. Chem. Fundam. 3, 123 (1964).

[19] N. Wakao and J. M. Smith, Chem. Eng. Sci. 17, 825 (1962).

[20] R. N. Foster and J. B. Butt, AIChE J. 12, 180 (1966).

[21] J. Szekely and J. W. Evans, Chem. Eng. Sci. 25, 1091 (1970).

[22] R. Mann and G. Thomson, Chem. Eng. Sci. 42, 555 (1987).

[23] V. N. Burganos and S. V. Sotirchos, AIChE J. 33, 1678 (1987).

[24] J. Wood and L. F. Gladden, Chem. Eng. Sci. 57, 3047 (2002).

[25] J. Wood and L. F. Gladden, Chem. Eng. Sci. 57, 3033 (2002).

[26] P. Rajniak and R. T. Yang, AIChE J. 42, 319 (1996).

[27] V. Novak, P. Koci, F. Štěpánek, and M. Marek, Ind. Eng. Chem. Res. 50, 12904 (2011). 
[28] F. Dorai, C. Moura Teixeira, M. Rolland, E. Climent, M. Marcoux, and A. Wachs, Chem. Eng. Sci. 129, 180 (2015).

[29] F. Larachi, R. Hannaoui, P. Horgue, F. Augier, Y. Haroun, S. Youssef, E. Rosenberg, M. Prat, and M. Quintard, Chem. Eng. J. 240, 290 (2014).

[30] V. Novak, F. Stepanek, P. Koci, M. Marek, and M. Kubicek, Chem. Eng. Sci. 65, 2352 (2010).

[31] M. J. Blunt, M. D. Jackson, M. Piri, and P. H. Valvatne, Adv. Water Resour. 25, 1069 (2002).

[32] G. T. Vladisavljević, I. Kobayashi, M. Nakajima, R. A. Williams, M. Shimizu, and T. Nakashima, J. Memb. Sci. 302, 243 (2007).

[33] Sinha, Halleck, and C.-Y. Wang, Electrochem. Solid-State Lett. 9, A344 (2006).

[34] C. A. Baldwin, A. J. Sederman, M. D. Mantle, P. Alexander, and L. F. Gladden, J. Colloid Interface Sci. 181, 79 (1996).

[35] A. R. Riyadh, T. Karsten, and S W Clinton, Soil Sci. Soc. Am. J. 67, 1687 (2003).

[36] J.-Y. Arns, V. Robins, A. P. Sheppard, R. M. Sok, W. V. Pinczewski, and M. A. Knackstedt, Transp. Porous Media 55, 21 (2004).

[37] H. Dong and M. J. Blunt, Phys. Rev. E 80, 1 (2009).

[38] D. Silin and T. Patzek, Physica A 371, 336 (2006).

[39] F. A. L. Dullien, Fluid Transport and Pore Structure, 2nd ed. (Academic Press, San Diego, 1992).

[40] J. F. Richardson and W. N. Zaki, Trans. Inst. Chem. Eng. 32, 35 (1954).

[41] P. N. Sharratt and R. Mann, Chem. Eng. Sci. 42, 1565 (1987).

[42] G. S. Armatas, Chem. Eng. Sci. 61, 4662 (2006). 
[43] M. P. Hollewand and L. F. Gladden, Chem. Eng. Sci. 47, 2757 (1992).

[44] M. M. Mezedur, M. Kaviany, and W. Moore, AIChE J. 48, 15 (2002).

[45] M. P. Hollewand and L. F. Gladden, Chem. Eng. Sience 47, 1761 (1992).

[46] B. B. Mandelbrot, The Fractal Geometry of Nature, 2nd ed. (Freeman, San Francisco, 1983).

[47] D. Avnir, The Fractal Approach to Heterogeneous Chemistry (John Wiley \& Sons, Inc., Chichester, 1989).

[48] S. Havlin and D. Ben-Avraham, Adv. Phys. 51, 187 (2002).

[49] M.-O. Coppens and G. F. Froment, Chem. Eng. Sci. 50, 1013 (1995).

[50] M.-O. Coppens and G. F. Froment, Chem. Eng. Sci. 50, 1027 (1995).

[51] M.-O. Coppens, Catal. Today 53, 225 (1999).

[52] M.-O. Coppens and G. F. Froment, Chem. Eng. Sci. 49, 4897 (1994).

[53] P. Trogadas, V. Ramani, P. Strasser, T. F. Fuller, and M.-O. Coppens, Angew. Chemie Int. Ed. 55, 122 (2016).

[54] P. Trogadas, M. M. Nigra, and M.-O. Coppens, New J. Chem. 40, 4016 (2016).

[55] M.-O. Coppens, Curr. Opin. Chem. Eng. 1, 281 (2012).

[56] M.-O. Coppens, in Multiscale Methods Multiscale Methods Bridg. Scales Sci. Eng., edited by J. Fish (Oxford University Press, New York, 2010), pp. 536-559.

[57] S. Weiner and H. D. Wagner, Annu. Rev. Mater. Sci. 28, 271 (1998).

[58] J. Y. Rho, L. Kuhn-Spearing, and P. Zioupos, Med. Eng. Phys. 20, 92 (1998).

[59] P. Fratzl and R. Weinkamer, Prog. Mater. Sci. 52, 1263 (2007).

[60] http://www.gla.ac.uk/ibls/US/fab/tutorial/generic/bone2.html (n.d.).

[61] Http://juanribon.com/design/lung-Cancer-Diagram.php (n.d.). 
[62] https://3dprint.com/7729/3d-Print-Organs-Vascular/ (n.d.).

[63] http://earthobservatory.nasa.gov/IOTD/view.php?id=2704 (n.d.).

[64] E. R. Weibel, Morphometry of the Human Lung (Springer, Berlin, 1963).

[65] E. R. Weibel, The Pathway for Oxygen (Harvard University Press, Cambridge, MA., 1984).

[66] S. Gheorghiu, S. Kjelstrup, P. Pfeifer, and M.-O. Coppens, in Fractals Biol. Med., edited by T. F. Nonnenmacher, G. A. Losa, and E. R. Weibel (Springer, Birkhäuser, 2005), pp. $31-42$.

[67] C. Hou, S. Gheorghiu, M.-O. Coppens, V. H. Huxley, and P. Pfeifer, in Fractals Biol. Med., edited by T. F. Nonnenmacher, G. A. Losa, and E. R. Weibel (Springer, Birkhäuser, 2005), pp. 17-30.

[68] E. R. Weibel, Am. J. Physiol. 261, L361 (1991).

[69] C. D. Murray, Proc. Natl. Acad. Sci. U. S. A. 12, 207 (1926).

[70] C. D. Murray, Proc. Natl. Acad. Sci. U. S. A. 12, 299 (1926).

[71] F. J. Keil and C. Rieckmann, Chem. Eng. Sci. 54, 3485 (1994).

[72] S. van Donk, A. H. Janssen, J. H. Bitter, and K. P. de Jong, Catal. Rev. Eng. 45, 297 (2003).

[73] S. Gheorghiu and M.-O. Coppens, AIChE J. 50, 812 (2004).

[74] G. Wang, E. Johannessen, C. R. Kleijn, S. W. de Leeuw, and M.-O. Coppens, Chem. Eng. Sci. 62, 5110 (2007).

[75] G. Wang and M.-O. Coppens, Chem. Eng. Sci. 65, 2344 (2010).

[76] G. Wang and M.-O. Coppens, Ind. Eng. Chem. Res. 47, 3847 (2008).

[77] E. Johannessen, G. Wang, and M.-O. Coppens, Ind. Eng. Chem. Res. 46, 4245 (2007). 
[78] S. M. Rao and M.-O. Coppens, Chem. Eng. Sci. 83, 66 (2012).

[79] S. M. Rao and M.-O. Coppens, Ind. Eng. Chem. Res. 49, 11087 (2010).

[80] J. Wang, J. C. Groen, W. Yue, W. Zhou, and M.-O. Coppens, J. Mater. Chem. 18, 468 (2008).

[81] J. Wang, W. Yue, W. Zhou, and M.-O. Coppens, Microporous Mesoporous Mater. 120, 19 (2009).

[82] J. Kärger and S. Vasenkov, Microporous Mesoporous Mater. 85, 195 (2005).

[83] G. Ye, X. Duan, K. Zhu, X. Zhou, M.-O. Coppens, and W. Yuan, Chem. Eng. Sci. 132, $108(2015)$.

[84] F. J. Keil and C. Rieckmann, Hungarian J. Ind. Chem. 21, 277 (1993).

[85] C. Rieckmann, T. Duren, and F. J. Keil, Hungarian J. Ind. Chem. 25, 137 (1997).

[86] G. Ye, X. Zhou, M.-O. Coppens, and W. Yuan, AIChE J. 62, 451 (2016).

[87] G. Ye, X. Zhou, M.-O. Coppens, J. Zhou, and W. Yuan, AIChE J. 63, 78 (2017).

[88] W. H. J. Hogarth and J. B. Benziger, J. Power Sources 159, 968 (2006).

[89] J. Larminie and A. Dicks, Fuel Cell Systems Explained, 2nd ed. (John Wiley \& Sons, Inc., Chichester, 2003).

[90] S. Kjelstrup, M.-O. Coppens, J. G. Pharoah, and P. Pfeifer, Energy and Fuels 24, 5097 (2010).

[91] J. P. Kloess, X. Wang, J. Liu, Z. Shi, and L. Guessous, J. Power Sources 188, 132 (2009).

[92] R. Roshandel, F. Arbabi, and G. K. Moghaddam, Renew. Energy 41, 86 (2012).

[93] A. Arvay, J. French, J. C. Wang, X. H. Peng, and A. M. Kannan, Int. J. Hydrogen Energy 38, 3717 (2013).

[94] K. A. McCulloh, J. S. Sperry, and R. A. Frederick, Nature 421, 939 (2003). 
[95] P. Domachuk, K. Tsioris, F. G. Omenetto, and D. L. Kaplan, Adv. Mater. 22, 249 (2010).

[96] T. F. Sherman, J. Gen. Physiol. 78, 431 (1981).

[97] B. Ramos-Alvarado, A. Hernandez-Guerrero, F. Elizalde-Blancas, and M. W. Ellis, Int. J. Hydrogen Energy 36, 12965 (2011).

[98] J. Marquis and M.-O. Coppens, Chem. Eng. Sci. 102, 151 (2013). 